\title{
Impact of spar-nacelle-blade coupling on the edgewise response of floating offshore wind turbines
}

\author{
Van-Nguyen Dinh ${ }^{1 a}$, Biswajit Basu ${ }^{* 1}$ and Søren R.K. Nielsen ${ }^{2 b}$ \\ ${ }^{1}$ School of Engineering, Trinity College Dublin, Dublin, Ireland \\ ${ }^{2}$ Department of Civil Engineering, Aalborg University, Aalborg, Denmark
}

(Received June 12, 2013, Revised September 6, 2013, Accepted September 25, 2013)

\begin{abstract}
The impact of spar-nacelle-blade coupling on edgewise dynamic responses of spar-type floating wind turbines (S-FOWT) is investigated in this paper. Currently, this coupling is not considered explicitly by researchers. First of all, a coupled model of edgewise vibration of the S-FOWT considering the aerodynamic properties of the blade, variable mass and stiffness per unit length, gravity, the interactions among the blades, nacelle, spar and mooring system, the hydrodynamic effects, the restoring moment and the buoyancy force is proposed. The aerodynamic loads are combined of a steady wind (including the wind shear) and turbulence. Each blade is modeled as a cantilever beam vibrating in its fundamental mode. The mooring cables are modeled using an extended quasi-static method. The hydrodynamic effects calculated by using Morison's equation and strip theory consist of added mass, fluid inertia and viscous drag forces. The random sea state is simulated by superimposing a number of linear regular waves. The model shows that the vibration of the blades, nacelle, tower, and spar are coupled in all degrees of freedom and in all inertial, dissipative and elastic components. An uncoupled model of the S-FOWT is then formulated in which the blades and the nacelle are not coupled with the spar vibration. A 5MW S-FOWT is analyzed by using the two proposed models. In the no-wave sea, the coupling is found to contribute to spar responses only. When the wave loading is considered, the coupling is significant for the responses of both the nacelle and the spar.
\end{abstract}

Keywords: wind energy; floating offshore wind turbine; spar-type; edgewise vibration; blade-nacelle-spar coupling; hydrodynamic effects; Morison's equation

\section{Introduction}

The spar-floating offshore wind turbine (S-FOWT) seems to be the most suitable concept for deep-water areas because of its lower center of mass, small water plane area and deep-draft (Dinh and Basu 2013). For the design of an S-FOWT, the entire system composed of rotor, nacelle, tower, platform, mooring system; subjected to wind, wave and hydrodynamic loads should be analyzed using integrated models (Nielson et al. 2006). Several integrated modeling and analysis procedure for S-FOWTs have been proposed by integrating different codes. SIMO and RIFLEX

* Corresponding author, Professor, E-mail: basub@tcd.ie

${ }^{a}$ Marie Curie Postdoctoral Research Fellow, E-mail: nguyendv@tcd.ie

${ }^{\mathrm{b}}$ Professor, E-mail: srkn@civil.aau.dk

Copyright @ 2013 Techno-Press, Ltd.

http://www.techno-press.org/?journal=csm\&subpage=7

ISSN: 2234-2184 (Print), 2234-2192 (Online) 
codes were coupled to enable a FEM formulation and integrated dynamic analysis of the HYWIND S-FOWT (Nielsen et al. 2006). In the simulation of the HYWIND S-FOWT (Skaare et al. 2007), the hydrodynamic models were offered by SIMO, the structural modeling was accomplished by RIFLEX, the rotor and the nacelle were modeled by HAWC2, and the mooring lines and the platform were modeled in SIMO-RIFLEX. FAST/ADAMS ${ }^{\circledR}$ was coupled with AeroDyn-HydroDyn-WAMIT in an integrated modeling for S-FOWTs (Jonkman 2007). In the complimentary analysis of a S-FOWT (Karimirad and Moan 2011), the HAWC2 code was used for aero-hydro-elastics, the mooring system was modeled in SIMO-RIFLEX and applied as nonlinear spring stiffness in HAWC2 through a dynamic link library interface; the hydrodynamic loads in HAWC2 code were based on Morison's formula. The coupled wave- and wind-induced motions of a 5-MW S-FOWT in harsh and operational environmental conditions were analyzed by using the coupled SIMO-RIFLEX-HAWC2 codes (Karimirad and Moan 2012).

In spite of the availability of a large number of codes which can accurately and in detail analyze different elements and components of a floating offshore wind turbine, no single code exists to analyze the system considering implicitly the coupling between different elements. In the literature, formulation of mass, damping and stiffness matrices and the load vectors of S-FOWT systems have rarely been reported. The direct integration of codes conserves the system matrices and the load vectors of the blade, nacelle, tower and spar for each component of the codes though some effects may be transferred by iteration which can be computationally expensive and inconvenient. Sultania (2010) modeled an S-FOWT by direct assembly of the upper wind turbine system matrices and the lower platform system matrices whereas the blade-nacelle-spar coupling terms were excluded in the assembled matrices and load vectors. Solberg (2011) assumed both tower and spar to be rigid and combined them as a structure with new center of gravity whereas several components were not considered in that combined structure such as the rotor-nacelle, the coupling among the blades, the tower and the spar. No study exists currently which investigates how significant the effect of the coupling terms could be. Hence, the coupling of the blades and nacelle with the spar vibration and their impact on the response of S-FOWTs needs to be investigated.

Investigation on the impact of blade-nacelle-spar vibration couplings is proposed to be studied with simplified models to see if the impact is considerable and to identify which loading conditions are critical. Hardly any simplified models have been proposed by researchers for the analysis of FOWTs and the S-FOWT in particular. Qualitative analyses of FOWTs under aero-hydrodynamic loads were described (Henderson and Patel 2003). Newton-Euler equations combined with constraint conditions were employed to analyze a 2-MW downwind S-FOWT in steady wind and no waves (Matsukuma and Utsunomiya 2008). There is also a lack of simplified models which couple the blades-rotor-tower-spar-mooring of S-FOWT and account for its aerodynamic, hydrodynamic and restoring forces. Thus, developing such a simplified coupled model and investigating the impact of the coupling on the response of S-FOWTs is the focus of this paper. The simplified models could also be used for a preliminary design and analysis tool prior to the use of complete coupled codes which could be computationally expensive when available by modification/merging of a number existing complicated codes.

The paper aims to examine the coupling effects by developing a simplified discrete system model of the complete system. For the purpose of simplification, the proposed model in this paper can be limited to the main modes of blade vibration in a wind turbine. The edgewise vibrations of onshore wind turbines are shown to be lightly damped and can lead to violent vibrations and the first edgewise mode may exhibit a very low or even negative damping under certain conditions 
(Staino et al. 2012). The edgewise vibrations of onshore wind turbines were studied (Staino et al. 2012, Basu et al. 2012). This paper proposes a coupled model describing the dynamics of edgewise vibrations of S-FOWTs, considering the aerodynamic properties of the blade, variable mass and stiffness per unit length, the effect of centrifugal stiffening, gravity, hydrostatic restoring moment, buoyancy; and the interactions among the blades, nacelle, tower and the spar. Aerodynamic loads corresponding to a combination of steady wind including the wind shear, and the turbulent component are computed by applying the modified Blade Element Momentum (BEM) theory (Hansen 2003). Each blade is modeled as a Bernoulli-Euler cantilever beam vibrating in its fundamental mode. The mooring cables are modeled using an extended quasi-static method (Sannasiraj et al. 1998). The hydrodynamic effects are calculated by using Morison's equation and strip theory, and consist of added mass forces, fluid inertia forces and viscous drag forces. The random sea state is simulated by superimposing a number of linear regular waves generated from the Pierson-Moskowitz wave spectrum. An uncoupled model is considered where the terms that couple the blades and nacelle with the spar vibrations are excluded. Finally, a 5MW S-FOWT of NREL is analyzed by using the two proposed models. In the no-wave loading case, the blade-nacelle-spar coupling affects the spar responses only. Under wave loading, the impact of the coupling on the responses of both the nacelle and the spar is significant.

\section{Coupled model of spar-floating offshore wind turbine}

\subsection{Modeling}

The spar is assumed to be a rigid body due to its large cross section. The displacement and velocity of the FOWT system are assumed to be small. As the spar diameter is small compared to its draft and to the wavelength, Morison's equation can be used to calculate the hydrodynamic loads. The proposed coupled model of a three-bladed horizontal-axis S-FOWT is shown in Fig. 1 and the definition of the model parameters is given in Table 1. The coupled model aims at representing the edgewise vibration responses and the associated coupling of the blades with the motions of the tower/nacelle and the spar. In this model, the blades are modeled as Bernoulli-Euler cantilever beams of length ' $R$ ', with variable bending stiffness and variable mass per unit length $\mu(r)$ along the length. The blades rotate at a constant speed $\Omega(\mathrm{rad} / \mathrm{s})$ and the azimuthal angle $\Psi_{i}(t)$ of blade ' $i$ ' at the time instant ' $t$ ' is given by Eq. (1)

$$
\psi_{i}(t)=\psi_{i}(t)+(i-1) \frac{2 \pi}{3}, \quad \psi_{1}(t)=\Omega t, \quad i=1,2,3
$$

The tower is modeled as a single degree of freedom (SDOF) system with the generalized stiffness $k_{t}$. Only the fundamental mode of the blade is considered in this paper. As the axial deformations of the spar and the tower are neglected, and the roll displacement of the spar $\varphi_{\mathrm{G}}$ is small, the absolute vertical displacement of the nacelle is approximated as $\bar{v}_{\text {nac }}=v_{G}-h_{a}$ $\left(1-\cos \varphi_{G}\right) \approx v_{G}$. The absolute horizontal displacement of the nacelle $\bar{u}_{\text {nac }}$ is approximated in terms of its horizontal displacement relative to the spar $u_{\text {nac }}$, the spar sway displacement $u_{G}$ and the spar roll displacement $\varphi_{G}$ as $\bar{u}_{\text {nac }} \approx u_{\text {nac }}+u_{G}-h_{a} \varphi_{G}$ where $h_{a}$ is the vertical distance between the 
tower top and the center of gravity, $h_{a}=h_{t}+h_{G}$.

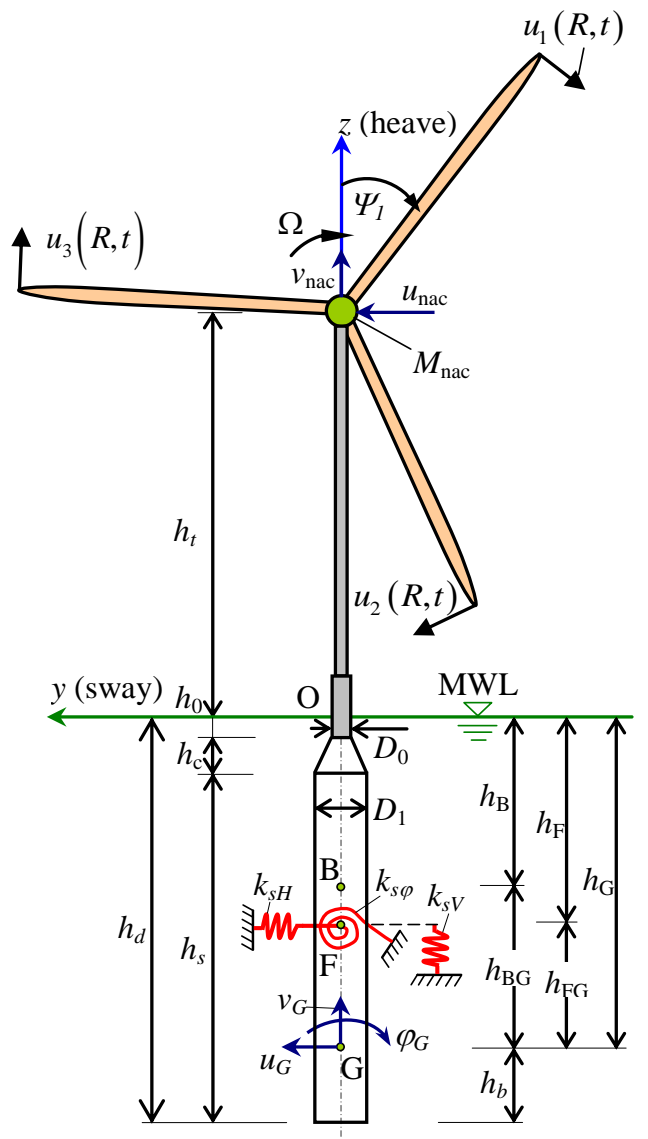

(a) Coupled model

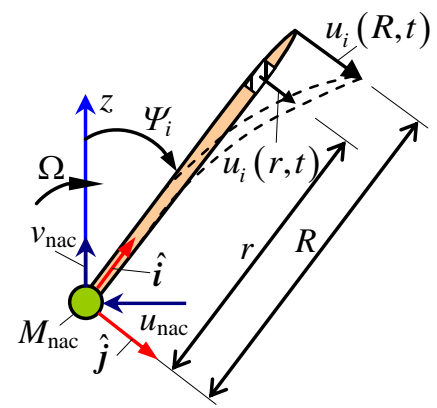

(b) Geometry of blade $i$

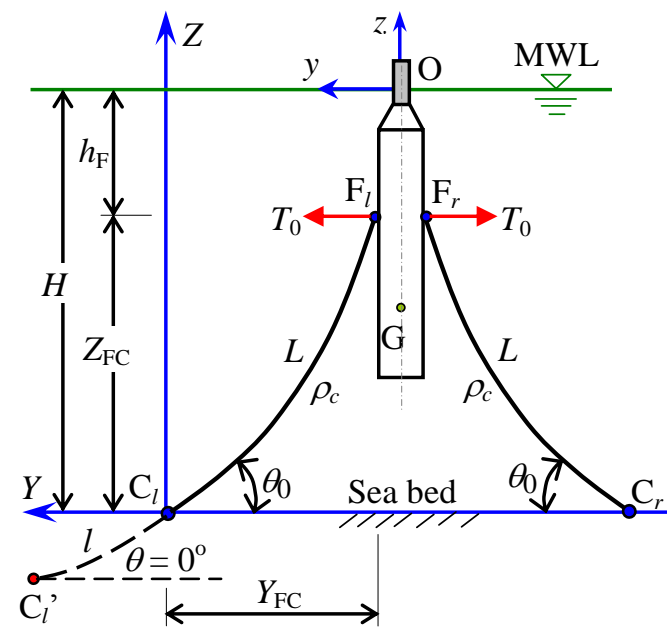

(c) Mooring model

Fig. 1 Edgewise model of S-FOWT

The vector of generalized coordinates of the systems is defined as

$$
\mathbf{q}(t)=\left\langle q_{1}(t) \quad q_{2}(t) \quad q_{3}(t) \quad q_{4}(t) \quad u_{G} \quad v_{G} \quad \varphi_{G}\right\rangle^{T}
$$


Table 1 Notations for edge-wise model of S-FOWT

\begin{tabular}{ll}
\hline \hline B = Center of buoyancy of the S-FOWT. & $M_{s}=$ Mass of the spar. \\
G = Center of gravity of the S-FOWT. & $M_{s}, I_{s}=$ Moment of inertia of the spar. \\
F = Fairlead position at spar centreline. & $\theta_{0}=$ Angle at the anchor points $\mathrm{C}_{l}$ and $\mathrm{C}_{r}$ \\
Oyz = Global axes, $O$ at mean water level (MWL) & $h_{t}=$ Tower top (yaw bearing) above MWL \\
$u_{G}, v_{G}, \varphi_{G}=$ Sway, heave and roll disp. at G. & $h_{0}=$ Depth to top of taper below MWL. \\
$k_{s H}, k_{s V}, k_{s \varphi}=$ Horizontal, vertical and roll stiffness of & $h_{c}=$ Height of spar taper \\
$\quad$ mooring system, respectively. & $h_{s}=$ Height of spar cylinder (from taper bottom to \\
$M_{c}=$ Mass of each mooring cable in water. & \multicolumn{1}{c}{ spar bottom) } \\
$M_{0}=$ Mass of nacelle + modal mass of tower & $h_{d}=$ Depth to spar bottom below MWL \\
$\rho_{c}=$ Cable mass per unit length. & $h_{G}=$ Depth of G below MWL. \\
$D_{0}=$ Diameter of spar above taper. & $h_{B}=$ Depth of B below MWL. \\
$\quad D_{1}=$ Diameter of spar below taper. &
\end{tabular}

where the degree of freedom $q_{i}(t), i=1,2,3$ relates to the fundamental edgewise mode of the blade " $i$ ". The variable $q_{4}(t)=u_{\text {nac }}(t)$ represents the motion of the nacelle in the rotor plane. The edgewise displacement along the blade $i$ can be approximated by using its fundamental edgewise mode shape $\phi_{1}(r)$ as $u_{i}(r, t)=\phi_{1}(r) q_{i}(t)$.

\subsection{System energy}

The absolute velocity vector of a point $r$ on the blade $i$ at time $t$ is written as (refer to Fig. 1(b))

$$
\mathbf{v}_{b i}(r, t)=\left[-\dot{\bar{u}}_{\text {nac }} \sin \psi_{i}+\dot{\bar{v}}_{\text {nac }} \cos \psi_{i}-\Omega u_{i}(r, t)\right] \hat{\mathbf{i}}+\left[-\dot{\bar{u}}_{\text {nac }} \cos \psi_{i}-\dot{\bar{v}}_{\text {nac }} \sin \psi_{i}+\dot{u}_{i}(r, t)+\Omega r\right] \hat{\mathbf{j}}
$$

where $\hat{\mathbf{i}}$ and $\hat{\mathbf{j}}$ are the unit vectors along and perpendicular to the blade center line, respectively.

The total kinetic energy of the whole S-FOWT system is expressed as

$$
T=\frac{1}{2} \sum_{i=1}^{3} \int_{0}^{R} \mu(r)\left|\mathbf{v}_{b i}\right|^{2}(r, t) d r+\frac{1}{2} M_{\text {nac }}\left(\dot{\bar{u}}_{\text {nac }}^{2}+\dot{\bar{v}}_{\text {nac }}^{2}\right)+\frac{1}{2} M_{s}\left(\dot{u}_{G}^{2}+\dot{v}_{G}^{2}\right)+\frac{1}{2} I_{s} \dot{\varphi}_{G}^{2}
$$

The total potential energy of whole S-FOWT system is expressed as

$$
V=\frac{1}{2} \sum_{i=1}^{3}\left(K_{e}+K_{w} \cos \psi_{i}+K_{g}\right) q_{i}^{2}+\frac{1}{2} k_{t} \bar{u}_{\mathrm{nac}}^{2}+\frac{1}{2} k_{s H} u_{G}^{2}+\frac{1}{2} k_{s V} v_{G}^{2}+\frac{1}{2} k_{s \varphi} \varphi_{G}^{2}
$$

where the parameter $K_{e}$ is the generalized elastic stiffness of the blade, $K_{e}=\omega_{b}^{2} m_{2}$ with $\omega_{b}$ being the fundamental natural frequency of the blade and $k_{t}$ is the modal stiffness of the tower. The term $K_{w}$ is 
the stiffness arising out of gravity effects, $K_{w}=-g \int_{0}^{R}\left[\int_{r}^{L} \mu(\xi) d \xi\right]\left[\phi_{1}^{\prime}\right]^{2} d r$. The term $K_{g}=\Omega^{2} K_{g, 0}$ is the geometrical stiffness due to centrifugal force on blade in which $K_{g, 0}=\int_{0}^{R}\left[\int_{r}^{L} \mu(\xi) \xi d \xi\right]\left[\phi_{1}^{\prime}\right]^{2} d r$. These blade-related parameters are defined in Staino et al. (2012).

\subsection{Generalized loads}

The virtual work done by external wind loads acting on the S-FOWT system is

$$
\delta W_{\text {wind }}=\sum_{i=1}^{3}\left(\int_{0}^{R} p_{i}(r, t) \phi_{1} d r \delta q_{i}-Q_{i} \delta \bar{u}_{\mathrm{nac}} \cos \psi_{i}-Q_{i} \delta \bar{v}_{\mathrm{nac}} \sin \psi_{i}\right)
$$

where $Q_{i}=\int_{0}^{R} p_{i}(r, t) d r$ is the total wind force acting on blade $i$ in the edgewise direction and $p_{i}(r, t)$ $(i=1,2,3)$ is the variable wind load intensity along the blade length in the edgewise direction calculated by using BEM theory (Hansen 2003, Staino et al. 2012). The wind load acting on the tower is neglected as it is small compared to the load on the rotor.

The virtual work done due to the gravity on the S-FOWT system is expressed as

$$
\delta W_{g}=g \sum_{i=1}^{3} \sin \psi_{i}\left[\int_{0}^{R} \mu(r) \phi_{1} d r \delta q_{i}-\int_{0}^{R} \mu(r) d r\left(\delta \bar{u}_{\mathrm{nac}} \cos \psi_{i}+\delta \bar{v}_{\mathrm{nac}} \sin \psi_{i}\right)\right]-M_{0} g \delta \bar{v}_{\mathrm{nac}}-M_{s} g \delta v_{G}
$$

\subsection{Euler-Lagrange equations and system matrices}

Substituting the system kinetic and potential energy into the Euler-Lagrange equations

$$
\frac{d}{d t}\left(\frac{\partial T}{\partial \dot{\mathbf{q}}}\right)-\frac{\partial T}{\partial \mathbf{q}}+\frac{\partial V}{\partial \mathbf{q}}=Q_{\mathrm{ext}}
$$

the mass, damping and stiffness matrices of the S-FOWT system can be obtained and are shown in Eqs. (9), (10) and (11), respectively.

$$
\mathbf{M}(t)=\left[\begin{array}{ccccccc}
m_{2} & 0 & 0 & -m_{1} \cos \psi_{1} & -m_{1} \cos \psi_{1} & -m_{1} \sin \psi_{1} & h_{a} m_{1} \cos \psi_{1} \\
0 & m_{2} & 0 & -m_{1} \cos \psi_{2} & -m_{1} \cos \psi_{2} & -m_{1} \sin \psi_{2} & h_{a} m_{1} \cos \psi_{2} \\
0 & 0 & m_{2} & -m_{1} \cos \psi_{3} & -m_{1} \cos \psi_{3} & -m_{1} \sin \psi_{3} & h_{a} m_{1} \cos \psi_{3} \\
-m_{1} \cos \psi_{1} & -m_{1} \cos \psi_{2} & -m_{1} \cos \psi_{3} & m_{4} & m_{4} & 0 & -h_{a} m_{4} \\
-m_{1} \cos \psi_{1} & -m_{1} \cos \psi_{2} & -m_{1} \cos \psi_{3} & m_{4} & m_{4}+M_{s} & 0 & -h_{a} m_{4} \\
-m_{1} \sin \psi_{1} & -m_{1} \sin \psi_{2} & -m_{1} \sin \psi_{3} & 0 & 0 & m_{4}+M_{s} & 0 \\
h_{a} m_{1} \cos \psi_{1} & h_{a} m_{1} \cos \psi_{2} & h_{a} m_{1} \cos \psi_{3} & -h_{a} m_{4} & -h_{a} m_{4} & 0 & I_{s}+h_{a}^{2} m_{4}
\end{array}\right]
$$




$$
\begin{aligned}
& \mathbf{C}(t)=\left[\begin{array}{ccccccc}
c_{b} & 0 & 0 & 0 & 0 & 0 & 0 \\
0 & c_{b} & 0 & 0 & 0 & 0 & 0 \\
0 & 0 & c_{b} & 0 & 0 & 0 & 0 \\
2 \Omega m_{1} \sin \psi_{1} & 2 \Omega m_{1} \sin \psi_{2} & 2 \Omega m_{1} \sin \psi_{3} & c_{4}+c_{t} & 0 & 0 & 0 \\
2 \Omega m_{1} \sin \psi_{1} & 2 \Omega m_{1} \sin \psi_{2} & 2 \Omega m_{1} \sin \psi_{3} & 0 & c_{u_{G}} & 0 & 0 \\
-2 \Omega m_{1} \cos \psi_{1} & -2 \Omega m_{1} \cos \psi_{2} & -2 \Omega m_{1} \cos \psi_{3} & 0 & 0 & c_{v_{G}} & 0 \\
-2 h_{a} \Omega m_{1} \sin \psi_{1} & -2 h_{a} \Omega m_{1} \sin \psi_{2} & -2 h_{a} \Omega m_{1} \sin \psi_{3} & 0 & 0 & 0 & c_{\varphi_{G}}
\end{array}\right] \\
& \mathbf{K}(t)=\left[\begin{array}{ccccccc}
k_{2}+K_{w} \cos \psi_{1} & 0 & 0 & 0 & 0 & 0 & 0 \\
0 & k_{2}+K_{w} \cos \psi_{2} & 0 & 0 & 0 & 0 & 0 \\
0 & 0 & k_{2}+K_{w} \cos \psi_{3} & 0 & 0 & 0 & 0 \\
\Omega^{2} m_{1} \cos \psi_{1} & \Omega^{2} m_{1} \cos \psi_{2} & \Omega^{2} m_{1} \cos \psi_{3} & k_{t} & k_{t} & 0 & -h_{a} k_{t} \\
\Omega^{2} m_{1} \cos \psi_{1} & \Omega^{2} m_{1} \cos \psi_{2} & \Omega^{2} m_{1} \cos \psi_{3} & k_{t} & k_{t}+k_{s H} & 0 & -h_{a} k_{t} \\
\Omega^{2} m_{1} \sin \psi_{1} & \Omega^{2} m_{1} \sin \psi_{2} & \Omega^{2} m_{1} \cos \psi_{3} & 0 & 0 & k_{s V} & 0 \\
-h_{a} \Omega^{2} m_{1} \cos \psi_{1} & -h_{a} \Omega^{2} m_{1} \cos \psi_{2} & -h_{a} \Omega^{2} m_{1} \cos \psi_{3} & -h_{a} k_{t} & -h_{a} k_{t} & 0 & h_{a}^{2} k_{t}+k_{s \varphi}
\end{array}\right]
\end{aligned}
$$

where

$$
m_{1}=\int_{0}^{R} \mu(r) \phi_{1} d r, \quad m_{2}=\int_{0}^{R} \mu(r) \phi_{1}^{2} d r, \quad m_{4}=3 \int_{0}^{R} \mu(r) d r+M_{0}, \quad k_{2}=K_{e}+\Omega^{2} K_{g}-\Omega^{2} m_{2}
$$

The terms $c_{b}$ and $c_{4}$ denote the structural and the aerodynamic damping associated with the blades and the nacelle, respectively. The term $c_{t}$ is the structural damping coefficient of the tower. The terms $c_{u_{G}}, c_{v_{G}}$ and $c_{\varphi_{G}}$ are respectively the structural damping coefficients in sway, heave and roll directions of the mooring system.

\subsection{Aerodynamic and gravitational loads}

Differentiating the virtual work done due to the wind load (given in Eq. (6)) with respect to the generalized coordinates, the generalized aerodynamic load vector is obtained as

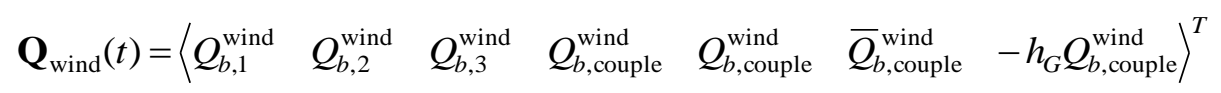

where the resultant aerodynamic load on blade $j$, and the aerodynamic loads coupling the blade edge-wise vibrations and the tower/spar sway and heave motions are, respectively

$$
Q_{b, i}^{\text {wind }}=\int_{0}^{R} p_{i}(r, t) \phi_{1} d r, \quad Q_{b, \text { couple }}^{\text {wind }}=-\sum_{i=1}^{3} \int_{0}^{R} p_{i}(r, t) d r \cos \psi_{i}, \quad \bar{Q}_{b, \text { couple }}^{\text {wind }}=-\sum_{i=1}^{3} \int_{0}^{R} p_{i}(r, t) d r \sin \psi_{i}
$$

in which the variable wind load intensity along the blade length in the edgewise direction, $p_{i}(x, t)(i=$ $1,2,3$ ) is calculated by using BEM theory (Hansen 2003, Staino et al. 2012). 
Differentiating the virtual work done due to the gravity (given in Eq. (7)), with respect to the generalized coordinates, the generalized gravitational load vector is obtained as

$$
\mathbf{Q}_{g}(t)=\left\langle\begin{array}{lllllll}
Q_{g 1} & Q_{g 2} & Q_{g 3} & 0 & 0 & Q_{g, v_{G}} & 0
\end{array}\right\rangle^{T}
$$

where the generalized gravitational loads on blade $i$ and on the spar are, respectively

$$
Q_{g i}=g m_{1} \sin \psi_{i}, \quad Q_{g, v_{G}}=-g\left[\sum_{i=1}^{3} \int_{0}^{R} \mu(r) d r \sin ^{2} \psi_{i}+M_{0}+M_{s}\right], i=1,2,3
$$

\section{Hydrostatic and restoring effects}

First of all, the initial depth of the top of taper below the mean water level (MWL) $h_{0}$ is re-calculated by using Archimedes's principle. The total weight of the wind turbine, platform and mooring cables in water is equal to the weight of the water volume displaced by the platform.

$$
h_{0}=\frac{1}{A_{s 0}}\left(\frac{M_{\text {all }}}{\rho_{w}}-V_{2}-V_{3}\right), \quad A_{s 0}=\frac{\pi D_{0}^{2}}{4}
$$

where $M_{\text {all }}$ is the total mass of the wind turbine, spar and the mooring cables in water, $A_{s 0}$ is the plane water area; $V_{2}$ and $V_{3}$ are the volume of water displaced by the taper part and by the main cylinder below the taper, respectively. The related depths such as $h_{d}, h_{\mathrm{F}}$ and $h_{\mathrm{G}}$ can then be re-calculated from $h_{0}$ and the fixed dimensions of the spar.

The vertical distance between the spar center of buoyancy B and center of gravity G is

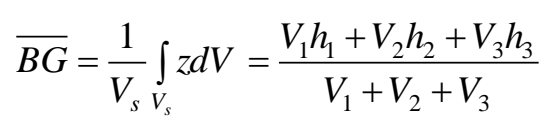

where $V_{1}$ is the volume of water displaced by the submersed part above the taper and $V_{s}=V_{1}+V_{2}+$ $V_{3}$. The vertical distances from $\mathrm{G}$ to the center of gravity of $V_{1}, V_{2}$ and $V_{3}$ are $h_{1}, h_{2}$ and $h_{3}$, respectively.

As the spar center of buoyancy B is always above its center of gravity $\mathrm{G}$, the downward distance from MWL to the center of buoyancy is $h_{B}=h_{G}-\overline{B G}$. The magnitude of the buoyancy force acting on the spar, and the vertical and roll restoring coefficients are, respectively given by

$$
F_{\text {buoy }}=\rho_{w} g V_{s}, \quad K_{V}^{\mathrm{res}}=\rho_{w} g A_{s 0}, \quad K_{\varphi}^{\mathrm{res}}=\left[\left(h_{B}-h_{G}\right) V_{s}+\frac{\pi D_{0}^{2}}{64}\right] \rho_{w} g
$$

where $\rho_{w}$ is the density of sea water. The roll restoring coefficient in Eq. (19) is obtained after evaluating the meta-centric height.

\section{Hydrodynamic effects}


The hydrodynamic effects on structures having a small dimension relative to the wavelength are widely expressed by using Morison's equation (Jonkman 2007, Karimirad and Moan 2012, Waris and Ishihara 2012, Abdel Raheem 2013). In this paper, the hydrodynamic effects on the spar expressed by using Morison's equation consists of (i) the forces related to the added mass associated with the spar accelerations $\ddot{u}_{s}$, (ii) the inertia forces associated with fluid accelerations, and (iii) the viscous drag forces proportional to the squared relative velocities of the spar and the fluid. In this section, those hydrodynamic forces are computed by using strip theory where the draft $h_{d}$ is divided into $N_{z}$ depth intervals $\Delta z_{i}$. Using the assumption of small roll velocity, the spar velocities in the horizontal and the vertical directions at a depth $z$ are respectively approximated as

$$
\dot{u}_{s}(z) \approx u_{G}-\left(z+h_{G}\right) \dot{\varphi}_{G}, \dot{v}_{s}(z) \approx \dot{v}_{G}
$$

Similar approximations of the spar accelerations in the horizontal and the vertical directions at a depth $z$ can be obtained by using an assumption of small roll acceleration $\ddot{\varphi}_{G}$.

\subsection{Added mass coefficients}

The hydrodynamic added mass coefficient matrix of the spar is expressed as

$$
\begin{gathered}
\mathbf{M}_{s}^{a}=\left[\begin{array}{ccc}
a_{y y} & 0 & a_{\varphi y} \\
0 & a_{z z} & 0 \\
a_{y \varphi} & 0 & a_{\varphi \varphi}
\end{array}\right] \text { where } a_{y y}=C_{M} \rho_{w} \frac{\pi}{4} \sum_{i=1}^{N_{z}} D^{2}\left(z_{i}\right) \Delta z_{i}, \quad a_{z z}=\rho_{w} \frac{\pi D_{1}^{3}}{12} \\
a_{y \varphi}=a_{\varphi y}=-C_{M} \rho_{w} \frac{\pi}{4} \sum_{i=1}^{N_{z}} D^{2}\left(z_{i}\right)\left(z_{i}+h_{G}\right) \Delta z_{i}, \quad a_{\varphi \varphi}=C_{M} \rho_{w} \frac{\pi}{4} \sum_{i=1}^{N_{z}} D^{2}\left(z_{i}\right)\left(z_{i}+h_{G}\right)^{2} \Delta z_{i}
\end{gathered}
$$

in which $a_{y y}$ is the added mass coefficient in sway motion due to sway acceleration, $a_{\varphi y}$ is the added mass coefficient in sway motion due to roll acceleration, $a_{\varphi \varphi}$ is the added mass roll moment due to spar roll acceleration, $C_{M}$ is the added mass coefficient $(=1.0$ for circular cylinder (Faltinsen 1990)) and $D\left(z_{i}\right)$ is the spar diameter at a discrete depth $z_{i}$. The vertical hydrodynamic added mass coefficient at the base of the spar $a_{z z}$ that cannot be predicted by using Morison's equation, is calculated by using the assumption that the volume of the added water under the spar is a half sphere (Haslum 1999, Waris and Ishihara 2012).

\subsection{Inertia forces of fluid particles}

When the waves are considered, the inertia forces are associated with fluid accelerations. These forces are larger at the depths closer to the surface. Hence, the total horizontal inertia fluid force should be evaluated by using the real shape of the spar as

$$
F_{y}^{f}(t)=\left(C_{M}+1\right) \rho_{w} \frac{\pi}{4} \sum_{i=1}^{N_{z}} D^{2}\left(z_{i}\right) \Delta z_{i} \ddot{u}_{f}\left(z_{i}, t\right)
$$

where $\ddot{u}_{f}\left(z_{i}, t\right)$ is the horizontal acceleration of fluid particles at depth $z_{i}$. The vertical inertia 
force due to vertical acceleration of fluid particles at the spar bottom $\ddot{v}_{f}\left(z=-h_{d}, t\right)$ is expressed as

$$
F_{z}^{f}(t)=\left(C_{M}+1\right) \rho_{w} A_{s 1} \ddot{v}_{f}\left(z=-h_{d}, t\right)
$$

where $A_{s 1}=\pi D_{1}^{2} / 4$. The fluid inertia forces in Eqs. (23) and (24) are zero in no-wave sea state.

\subsection{Viscous drag forces}

The viscous drag forces are dissipative and are resulted by the relative velocities of the spar and the fluid and exist regardless of the presence of the waves. The total horizontal drag force is

$$
F_{y}^{D f}=\frac{1}{2} C_{D}^{y} \rho_{w} \sum_{i=1}^{N_{z}} D\left(z_{i}\right) \mid \dot{\mathbf{q}}_{s f}^{n}\left(z_{i}, t\right)\left[\dot{u}_{f}\left(z_{i}, t\right)-\dot{u}_{G}+\dot{\varphi}_{G}\left(z_{i}+h_{G}\right)\right] \Delta z_{i}
$$

where $C_{D}^{y}$ is the hydrodynamic viscous drag coefficient of spar sides (= 0.6 for cylindrical object (Waris and Ishihara 2012)). The quantity $\left|\dot{\mathbf{q}}_{s f}^{n}\left(z_{i}, t\right)\right|$ is the amplitude of the relative normal velocity vector at $z_{i}$ and can be expressed as

$$
\left|\dot{\mathbf{q}}_{s f}^{n}(z, t)\right|=\sqrt{\left[\dot{u}_{f}(z, t)-\dot{u}_{G}+\dot{\varphi}_{G}\left(z+h_{G}\right)\right]^{2}+\left[\dot{v}_{f}(z, t)-\dot{v}_{G}\right]^{2}}
$$

where $\dot{u}_{f}\left(z_{i}, t\right)$ and $\dot{v}_{f}(z, t)$ are the fluid horizontal and vertical velocity, respectively.

The total vertical drag force normal to the spar bottom due to its heave velocity can be expressed by Eq. (27) (Kallesøe et al. 2011) with $C_{D}^{z}$ being the drag coefficient of heave motion

$$
F_{z}^{D f}=\frac{1}{2} C_{D}^{z} \rho_{w} A_{s 1}\left|\dot{\mathbf{q}}_{s f}^{n}\left(-h_{d}, t\right)\right|\left[\dot{v}_{f}\left(-h_{d}, t\right)-\dot{v}_{G}\right]
$$

\section{Uncoupled model}

In order to examine the impact of blade-nacelle-spar coupling on the responses of the S-FOWT, an uncoupled model is developed by excluding the coupling terms from the mass, damping and stiffness matrices of the S-FOWT in Eqs. (9)-(11) that yields

$$
\mathbf{M}^{\prime}(t)=\left[\begin{array}{ccccccc}
m_{2} & 0 & 0 & -m_{1} \cos \psi_{1} & 0 & 0 & 0 \\
0 & m_{2} & 0 & -m_{1} \cos \psi_{2} & 0 & 0 & 0 \\
0 & 0 & m_{2} & -m_{1} \cos \psi_{3} & 0 & 0 & 0 \\
-m_{1} \cos \psi_{1} & -m_{1} \cos \psi_{2} & -m_{1} \cos \psi_{3} & m_{4} & 0 & 0 & 0 \\
0 & 0 & 0 & 0 & M_{s} & 0 & -h_{G} M_{s} \\
0 & 0 & 0 & 0 & 0 & M_{s} & 0 \\
0 & 0 & 0 & 0 & -h_{G} M_{s} & 0 & I_{s}
\end{array}\right]
$$




$$
\begin{aligned}
\mathbf{C}^{\prime}(t) & =\left[\begin{array}{ccccccc}
c_{b} & 0 & 0 & 0 & 0 & 0 & 0 \\
0 & c_{b} & 0 & 0 & 0 & 0 & 0 \\
0 & 0 & c_{b} & 0 & 0 & 0 & 0 \\
2 \Omega m_{1} \sin \psi_{1} & 2 \Omega m_{1} \sin \psi_{2} & 2 \Omega m_{1} \sin \psi_{3} & c_{4}+c_{t} & 0 & 0 & 0 \\
0 & 0 & 0 & 0 & c_{u_{G}} & 0 & 0 \\
0 & 0 & 0 & 0 & 0 & c_{v_{G}} & 0 \\
0 & 0 & 0 & 0 & 0 & 0 & c_{\varphi_{G}}
\end{array}\right] \\
\mathbf{K}^{\prime}(t) & =\left[\begin{array}{ccccccc}
k_{2}+K_{w} \cos \psi_{1} & 0 & 0 & 0 & 0 & 0 & 0 \\
0 & k_{2}+K_{w} \cos \psi_{2} & 0 & 0 & 0 & 0 & 0 \\
0 & 0 & k_{2}+K_{w} \cos \psi_{3} & 0 & 0 & 0 & 0 \\
\Omega^{2} m_{1} \cos \psi_{1} & \Omega^{2} m_{1} \cos \psi_{2} & \Omega^{2} m_{1} \cos \psi_{3} & k_{t} & 0 & 0 & 0 \\
0 & 0 & 0 & 0 & k_{s H} & 0 & 0 \\
0 & 0 & 0 & 0 & 0 & k_{s V} & 0 \\
0 & 0 & 0 & 0 & 0 & 0 & k_{s \varphi}
\end{array}\right]
\end{aligned}
$$

In this uncoupled model, the aerodynamic loads coupling the blade edge-wise vibrations and the tower/spar sway, heave and roll motions in Eq. (13) are not considered, and the generalized aerodynamic load vector is reduced to

$$
\mathbf{Q}_{\text {wind }}^{\prime}(t)=\left\langle\begin{array}{lllllll}
Q_{b, 1}^{\text {wind }} & Q_{b, 2}^{\text {wind }} & Q_{b, 3}^{\text {wind }} & Q_{b, \text { couple }}^{\text {wind }} & 0 & 0 & 0
\end{array}\right\rangle^{T}
$$

The generalized gravitational load vector and the gravitational load on the spar are respectively given as

$$
\mathbf{Q}_{g}^{\prime}(t)=\left\langle\begin{array}{lllllll}
Q_{g 1} & Q_{g 2} & Q_{g 3} & 0 & 0 & Q_{g, v_{G}}^{\prime} & 0
\end{array}\right\rangle^{T}, \quad Q_{g, v_{G}}^{\prime}=-g\left(m_{4}+M_{s}\right)
$$

The spar-components in the mass, stiffness and damping matrices and load vector of this uncoupled model are the same as those in Sultania (2010).

\section{Equations of motion of the two S-FOWT models}

The equations of motion of the coupled model proposed in Section 2 and the uncoupled model proposed in Section 5 can be written in a compact form as

$$
\overline{\mathbf{M}}(t) \ddot{\mathbf{q}}(t)+\left[\overline{\mathbf{C}}(t)+\mathbf{C}^{\mathrm{drag}}(t)\right] \dot{\mathbf{q}}(t)+\overline{\mathbf{K}}(t) \mathbf{q}(t)=\overline{\mathbf{Q}}(t)
$$

For the coupled model 


$$
\begin{gathered}
\overline{\mathbf{M}}(t)=\mathbf{M}(t)+\mathbf{M}^{a}, \quad \overline{\mathbf{C}}(t)=\mathbf{C}(t)+\mathbf{C}^{\mathrm{mor}}, \quad \overline{\mathbf{K}}(t)=\mathbf{K}(t)+\mathbf{K}^{\mathrm{res}} \\
\overline{\mathbf{Q}}(t)=\mathbf{Q}_{g}(t)+\mathbf{Q}_{\mathrm{wind}}(t)+\mathbf{Q}_{\mathrm{buoy}}+\mathbf{Q}_{\mathrm{drag}}(t)+\mathbf{Q}_{f}(t)
\end{gathered}
$$

For the uncoupled model

$$
\begin{gathered}
\overline{\mathbf{M}}(t)=\mathbf{M}^{\prime}(t)+\mathbf{M}^{a}, \quad \overline{\mathbf{C}}(t)=\mathbf{C}^{\prime}(t)+\mathbf{C}^{\mathrm{mor}}, \quad \overline{\mathbf{K}}(t)=\mathbf{K}^{\prime}(t)+\mathbf{K}^{\mathrm{res}} \\
\overline{\mathbf{Q}}(t)=\mathbf{Q}_{g}^{\prime}(t)+\mathbf{Q}_{\text {wind }}^{\prime}(t)+\mathbf{Q}_{\mathrm{buoy}}+\mathbf{Q}_{\mathrm{drag}}(t)+\mathbf{Q}_{f}(t)
\end{gathered}
$$

For both the models, $\mathbf{C}^{\text {moor }}$ is the structural damping matrix of mooring systems,

$$
\mathbf{M}^{a}=\left[\begin{array}{cc}
\mathbf{0} & \mathbf{0} \\
\mathbf{0} & \mathbf{M}_{s}^{a}
\end{array}\right] \in \mathrm{R}^{7 \times 7} ; \quad \mathbf{K}^{\text {res }}=\operatorname{diag}\left\langle\begin{array}{cccc}
0 & 0 & K_{V}^{\text {res }} & K_{\varphi}^{\text {res }}
\end{array}\right\rangle \in \mathrm{R}^{7 \times 7}
$$

and the damping matrix $\mathbf{C}^{\text {drag }}(t)$ is defined from the drag forces (in Eqs. (25)-(27)) as

$$
\begin{aligned}
\mathbf{C}^{\mathrm{drag}}(t) & =\left[\begin{array}{cccc}
\mathbf{0} & \mathbf{0} & \mathbf{0} & \mathbf{0} \\
\mathbf{0} & C_{u u_{G}}^{\mathrm{drag}}(t) & 0 & C_{u \varphi_{G}}^{\mathrm{drag}}(t) \\
\mathbf{0} & 0 & C_{v v_{G}}^{\mathrm{drag}}(t) & 0 \\
\mathbf{0} & 0 & 0 & 0
\end{array}\right] \in \mathrm{R}^{7 \times 7} \text { where } C_{v v_{G}}^{\mathrm{drag}}(t)=\frac{1}{2} C_{D}^{z} \rho_{w} A_{s 1}\left|\dot{\mathbf{q}}_{s f}^{n}\left(-h_{d}, t\right)\right| \\
C_{u u_{G}}^{\mathrm{drag}} & =\frac{1}{2} C_{D}^{y} \rho_{w} \sum_{i=1}^{N_{z}} D\left(z_{i}\right) \Delta z_{i}\left|\dot{\mathbf{q}}_{s f}^{n}\left(z_{i}, t\right)\right|, \quad C_{u \varphi_{G}}^{\mathrm{drag}}=-\frac{1}{2} C_{D}^{y} \rho_{w} \sum_{i=1}^{N_{z}} D\left(z_{i}\right)\left(z_{i}+h_{G}\right) \Delta z_{i}\left|\dot{\mathbf{q}}_{s f}^{n}\left(z_{i}, t\right)\right|
\end{aligned}
$$

In Eqs. (35) and (37), the drag force vector is

$$
\begin{gathered}
\left.\mathbf{Q}_{\mathrm{drag}}(t)=\begin{array}{ccccccc}
0 & 0 & 0 & 0 & F_{y}^{D} & F_{z}^{D} & 0
\end{array}\right)^{T} \\
F_{y}^{D}=\frac{1}{2} C_{D}^{y} \rho_{w} \sum_{i=1}^{N_{z}} D\left(z_{i}\right) \Delta z_{i}\left|\dot{\mathbf{q}}_{s f}^{n}\left(z_{i}, t\right)\right| \dot{u}_{f}\left(z_{i}, t\right), \quad F_{z}^{D f}=\frac{1}{2} C_{D}^{z} \rho_{w} A_{s 1}\left|\dot{\mathbf{q}}_{s f}^{n}\left(-h_{d}, t\right)\right| \dot{v}_{f}\left(-h_{d}, t\right)
\end{gathered}
$$

and the inertia force vector due to fluid particle accelerations is

$$
\mathbf{Q}_{f}(t)=\left\langle\begin{array}{llllllll}
0 & 0 & 0 & 0 & F_{y}^{f} & F_{z}^{f} & 0
\end{array}\right\rangle^{T}
$$

where $F_{y}^{f}$ and $F_{z}^{f}$ are defined in Eqs. (23) and (24), respectively.

Let $\mathbf{X}_{1}=\mathbf{q}(t)$ and $\mathbf{X}_{2}=\dot{\mathbf{q}}(t)$, the equations of motion, Eq. (33) can be expressed in a solvable equations of space-state form as 


$$
\left\{\begin{array}{l}
\dot{\mathbf{X}}_{1} \\
\dot{\mathbf{X}}_{2}
\end{array}\right\}=\left[\begin{array}{cc}
\mathbf{0}_{7 \times 7} & \mathbf{I}_{7 \times 7} \\
-\overline{\mathbf{M}}^{-1} \overline{\mathbf{K}} & -\overline{\mathbf{M}}^{-1}\left(\overline{\mathbf{C}}(t)+\mathbf{C}^{\mathrm{drag}}(t)\right)
\end{array}\right]\left\{\begin{array}{l}
\mathbf{X}_{1} \\
\mathbf{X}_{2}
\end{array}\right\}+\left[\begin{array}{c}
\mathbf{0}_{7 \times 7} \\
\overline{\mathbf{M}}^{-1}
\end{array}\right]\left\{\begin{array}{c}
\mathbf{0}_{7 \times 1} \\
\overline{\mathbf{Q}}(t)
\end{array}\right\}
$$

\section{Model of the mooring cable}

The extended quasi-static model of mooring cable that has been applied into moored floating breakwaters (Sannasiraj et al. 1998) is used in this paper. In this model, the cable is assumed to be perfectly flexible, inextensible, and heavy. The linearized stiffness coefficients are evaluated from the cable mass per unit length of the mooring line $\rho_{c}$, the initial horizontal tension $T_{0}$ which is assumed to be constant along the cable length, the vertical coordinate of the fairlead $F$ with respect to the anchor point $Z_{\mathrm{FC}}$, the length $L$ and the angle at the anchor point $\theta_{0}$ as shown in Fig. 1(c). Neglecting the coupling between vertical and horizontal stiffness, the horizontal, vertical and rolling stiffness of the mooring system are respectively expressed as (Sannasiraj et al. 1998)

$$
\begin{gathered}
k_{s H}=\frac{w}{T_{0}}\left(\frac{L^{\prime} T_{C}-l T_{F}}{T_{F}-T_{C}}\right) k_{H V}, \quad k_{s V}=\frac{w}{T_{0}}\left(\frac{T_{F} T_{C}}{T_{F}-T_{C}}\right)\left[\frac{Y_{F C}}{T_{0}}-\left(\frac{L^{\prime} T_{C}-l T_{F}}{T_{F} T_{C}}\right)\right] k_{H V} \\
k_{s \varphi}=\left\{\begin{array}{l}
\frac{w\left(z_{F}-z_{G}\right)^{2}}{T_{0}}\left(\frac{L^{\prime} T_{C}-l T_{F}}{T_{F}-T_{C}}\right)+\frac{w\left(y_{G}-y_{F}\right)^{2}}{T_{0}}\left(\frac{T_{F} T_{C}}{T_{F}-T_{C}}\right)\left[\frac{Y_{F C}}{T_{0}}-\left(\frac{L^{\prime} T_{C}-l T_{F}}{T_{F} T_{C}}\right)\right] k_{H V} \\
+2\left(y_{G}-y_{F}\right)\left(z_{F}-z_{G}\right)
\end{array}\right.
\end{gathered}
$$

where $w$ is the weight per unit length of the cable in water, $l$ is the imaginary extension length to make a zero contact angle (Jain 1980). The term $Z_{\mathrm{FC}}$ is the vertical distance between the fairlead $\mathrm{F}$ and the imaginary anchor $\mathrm{C}^{\prime}, L$ is the real length of the cable and $L^{\prime}=L+l$ is its total length. The notations $Y_{F C}$, and $Y_{F C}$ are the horizontal distances between the fairlead $\mathrm{F}$ and the imaginary anchor $\mathrm{C}^{\prime}$ and the real anchor $\mathrm{C}$, respectively. The initial cable tensions at the seabed contact point and at the fairlead are denoted by $T_{C}$ and $T_{F}$, respectively. The parameters in Eqs. (45) and (46) are defined by Jain (1980).

\section{Model of ocean random waves}

The physical sea state of ocean waves is random. The random sea state is described in this paper by superimposing a number of linear regular waves with different heights, frequencies and phase angles. The surface elevation $\eta$ can be expressed as (Faltinsen 1990)

$$
\eta(y, t)=\sum_{j=1}^{N_{\omega}} A_{j} \sin \left(\omega_{j} t-k_{j} y+\varepsilon_{j}\right)
$$

where $A_{j}, \omega_{j}, k_{j}, \varepsilon_{j}$ are the wave amplitude, circular frequency, wave number and random phase 
angle of wave component number $j$, respectively. The random phase angles $\varepsilon_{j}$ are uniformly distributed between 0 and $2 \pi$. The parameters $\omega_{j}$ and $k_{j}$ are related by the approximated dispersion relationship $k_{j}=\omega_{j}^{2} / g$ for deep water or by the exact linear dispersion relationship $k_{j} \tanh \left(k_{j} H\right)$ $=\omega_{j}^{2} / g$ for any water depth (Sarpkaya and Isaacson 1981).

The simulation formulas for fluid velocity and acceleration in infinite water depth (Faltinsen 1990) can be extended to the more general case of finite water depth. The horizontal and the vertical velocity, and the horizontal and the vertical acceleration of fluid particles at a depth of $z$ (origin at the MWL and positive upward) and horizontal position $y$ are respectively given by

$$
\begin{aligned}
& \dot{u}_{f}(y, z, t)=\sum_{j=1}^{N_{\omega}} \omega_{j} A_{j} \frac{\cosh k_{j}(z+H)}{\sinh k_{j} H} \sin \theta_{j}(y, t) \\
& \dot{v}_{f}(y, z, t)=\sum_{j=1}^{N_{\omega}} \omega_{j} A_{j} \frac{\sinh k_{j}(z+H)}{\sinh k_{j} H} \sin \theta_{j}(y, t) \\
& \ddot{u}_{f}(y, z, t)=\sum_{j=1}^{N_{\omega}} \omega_{j}^{2} A_{j} \frac{\cosh k_{j}(z+H)}{\sinh k_{j} H} \sin \theta_{j}(y, t) \\
& \ddot{v}_{f}(y, z, t)=-\sum_{j=1}^{N_{\omega}} \omega_{j}^{2} A_{j} \frac{\sinh k_{j}(z+H)}{\sinh k_{j} H} \sin \theta_{j}(y, t)
\end{aligned}
$$

where the wave amplitude $A_{j}$ can be expressed by using a wave spectrum $S(\omega)$ as

$$
A_{j}=\sqrt{2 S\left(\omega_{j}\right) \Delta \omega}
$$

in which $\Delta \omega$ is a constant difference between successive frequencies. The parameter $\theta_{j}$ is defined as equal to $\omega_{j} t-k_{j} y+\varepsilon_{j}$. The Pierson-Moskowitz (PM) wave spectrum defined in the IEC 61400-3 (2006) design standard for offshore wind turbines and routinely used to describe the statistical properties of fully developed seas (Jonkman 2007) is used in this paper. The one-sided PM spectrum has a form of

$$
S(\omega)=\frac{1}{2 \pi} \frac{5}{16} H_{s w}^{2} T_{p w} c_{\omega}^{-5} \exp \left(-\frac{5}{4} c_{\omega}^{-4}\right)
$$

where $c_{\omega}=\omega T_{p w} /(2 \pi), H_{s w}$ is the significant wave height and $T_{p w}$ is the spectral period; their units must be meter and second, respectively. There is a possibility of correlating wind and wave loading conditions on offshore wind turbines (Colwell and Basu 2009). The wind and wave states obtained from simultaneous wind and wave measurements at Statfjord site in the northern North Sea (Johannessen et al. 2001) and used as an operating condition (Karimirad and Moan 2012) are therefore employed in the numerical examples. 


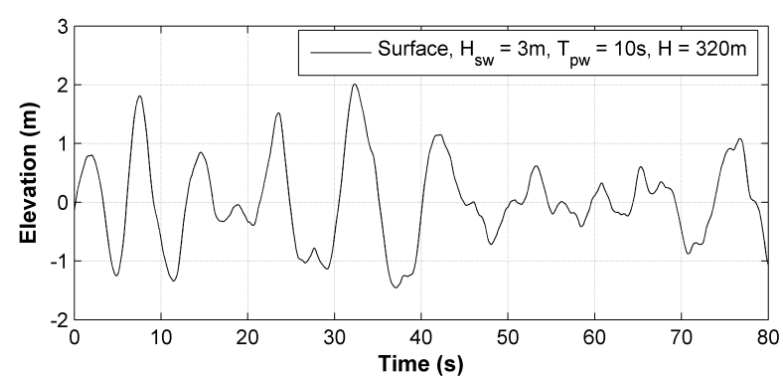

(a) Surface elevation time history

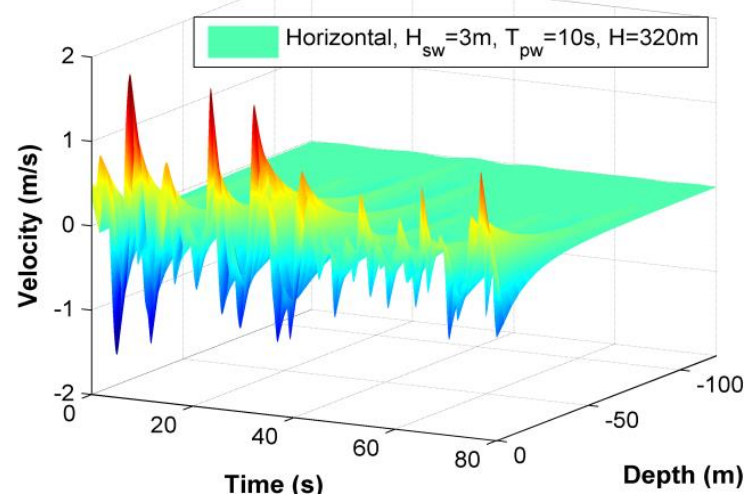

(c) Horizontal velocity in time-depth axes

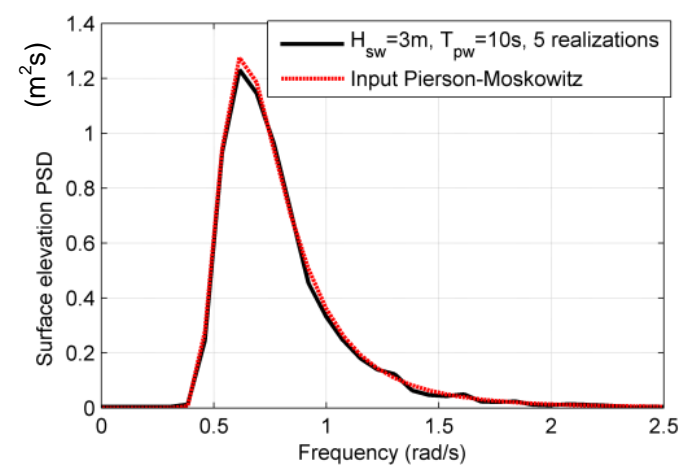

(b) Surface elevation PSD

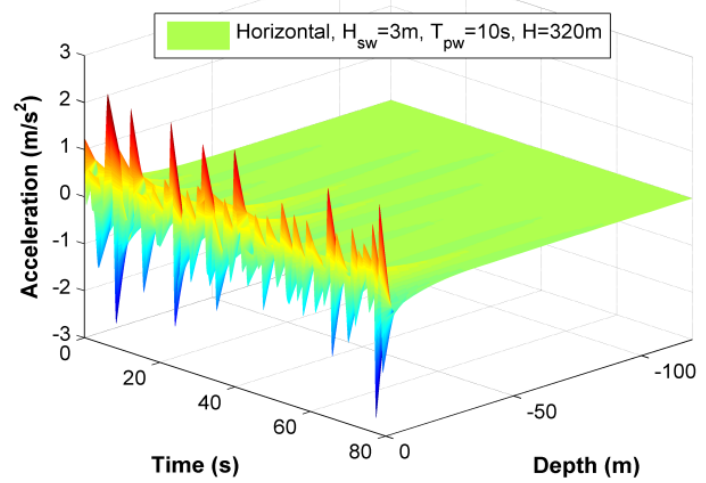

(d) Horizontal acceleration in time-depth axes

Fig. 2 Simulated sea profiles

\section{Numerical examples}

The examples assume the properties of the floating platform, mooring systems and tower of the OC3 S-FOWT (Jonkman 2010) with $h_{t}=87.6 \mathrm{~m}, h_{0}=4 \mathrm{~m}, h_{c}=8 \mathrm{~m}, h_{d}=120 \mathrm{~m}, h_{F}=70 \mathrm{~m}, h_{\mathrm{G}}=$ $89.9155 \mathrm{~m}, D_{0}=6.5 \mathrm{~m}, D_{1}=9.4 \mathrm{~m}, M_{s}=7466.33 \times 10^{3} \mathrm{~kg}, I_{s}=4229.23 \times 10^{6} \mathrm{~kg} . \mathrm{m}^{2}, L=902.2 \mathrm{~m}, \rho_{c}=$ $77.7066 \mathrm{~kg} / \mathrm{m}, \theta_{0}=0^{\circ}, T_{0}=100,000 \mathrm{~N}, \rho_{w}=1025 \mathrm{~kg} / \mathrm{m}^{3}$ and $H=320 \mathrm{~m}$. The calculated values of $h_{0}$ by using Eq. (22) and $h_{B}$ by using Eq. (24) are $4.85 \mathrm{~m}$ and $62.69 \mathrm{~m}$, respectively. The aerodynamic, blade, hub and nacelle properties of the NREL 5-MW baseline HAWT (Jonkman et al. 2009) are used with $R=61.5 \mathrm{~m}$, the cut-in and with rated rotor speeds as $6.9 \mathrm{rpm}$ and $12.1 \mathrm{rpm}$, respectively. The mass of each blade, hub mass, nacelle mass, and tower integrated mass are $17.74 \times 10^{3} \mathrm{~kg}$, $56.78 \times 10^{3} \mathrm{~kg}, 240 \times 10^{3} \mathrm{~kg}$, and $249.718 \times 10^{3} \mathrm{~kg}$, respectively. The fundamental frequencies of the blade and the tower are $6.81 \mathrm{rad} / \mathrm{s}$ and $2.87 \mathrm{rad} / \mathrm{s}$ respectively. The modal structural damping ratios of the blade and the tower are taken as $0.48 \%$ and $1 \%$, respectively (Jonkman et al. 2009). The drag coefficient of heave motion $C_{D}^{z}=20$ (Kallesøe et al. 2011) is initially used but the heave displacement of the spar is unrealistically damped out after a few vibration cycles. Hence a smaller value of $C_{D}^{z}=2$ is assumed and used in this paper. 


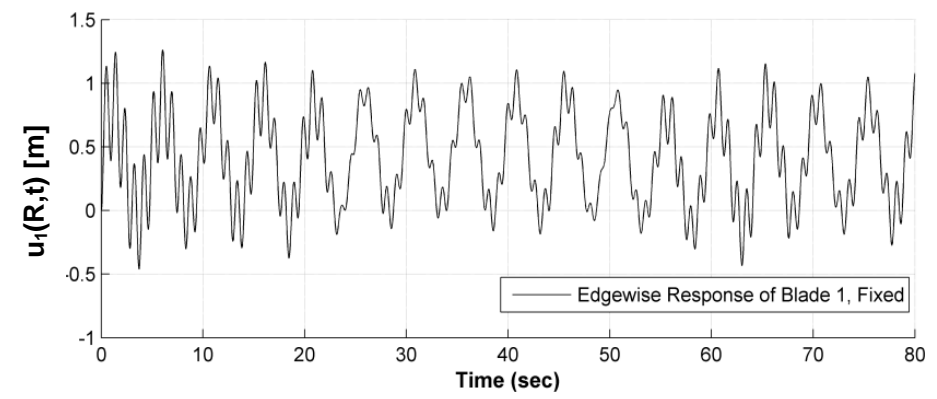

(a) Blade 1, time history

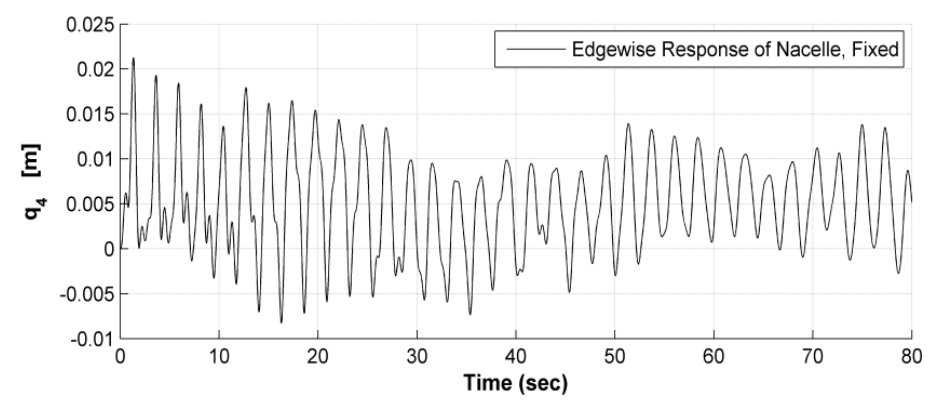

(c) Nacelle, time history

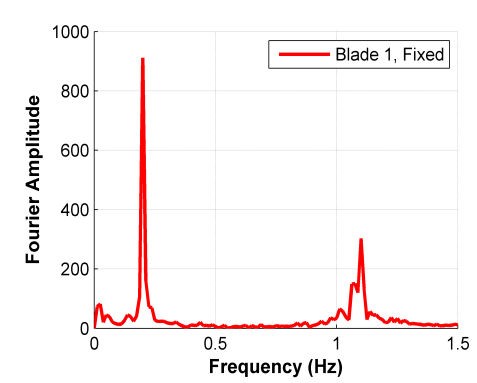

(b) Blade 1, FFT

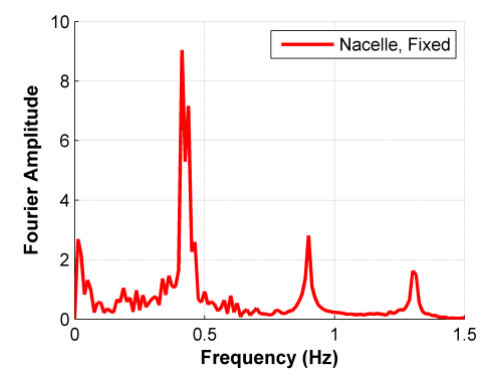

(d) Nacelle, FFT

Fig. 3 Displacements of a 5MW fixed-base WT

The wind and wave states used in the numerical examples are assumed to be at operating condition (Karimirad and Moan 2012). The mean wind speed at the top of the tower is assumed to be at the rated wind speed of $12 \mathrm{~m} / \mathrm{s}$. The turbulence intensity is $15 \%$, the significant wave height $H_{s w}$ is $3 \mathrm{~m}$ and the wave peak period $T_{p w}$ is $10 \mathrm{~s}$. To simulate the sea profiles by using Eqs. (47)-(51) and to calculate the added mass coefficients, drag forces and fluid inertia forces, the draft $h_{d}$ is divided into 30 intervals of $\Delta z=4 \mathrm{~m}$ along the depth, and the unrealistic high frequency components of waves are filtered out. The total simulation time, time step interval and the frequency interval are $80 \mathrm{~s}, 0.02 \mathrm{~s}$ and $0.0767 \mathrm{rad} / \mathrm{s}$ respectively. The simulated sea surface elevation and the 3-D profiles of horizontal velocity and acceleration are shown in Fig. 2. The 3-D sea profiles show that the sea responses are largest at surface level and reduce with the increasing depth. These are required for the computation of drag and fluid inertia forces by integrating over the depth.

Five cases are analyzed, namely (i) fixed-base wind turbine (FBWT) having the same aerodynamic loads and properties of blades, nacelle and tower as those of the OC3 S-FOWT, (ii) S-FOWT by using uncoupled model in no-wave sea, (iii) S-FOWT by using coupled model in no-wave sea, (iv) S-FOWT by using uncoupled model with waves, and (v) S-FOWT by using coupled model with waves. The displacements of blade 1 and nacelle for case (i) are shown in Fig. 3. The displacements of blade 1, nacelle and spar heave for case (ii) are shown in Fig. 4. In case (ii), the spar sway and roll displacement are almost zero. Figs. 5-7 show the displacements of blade 1, nacelle and spar sway, heave and roll for cases (iii), (iv) and (v) respectively.

\subsection{Response comparisons of a fixed-base WT and a S-FOWT in no-wave sea}




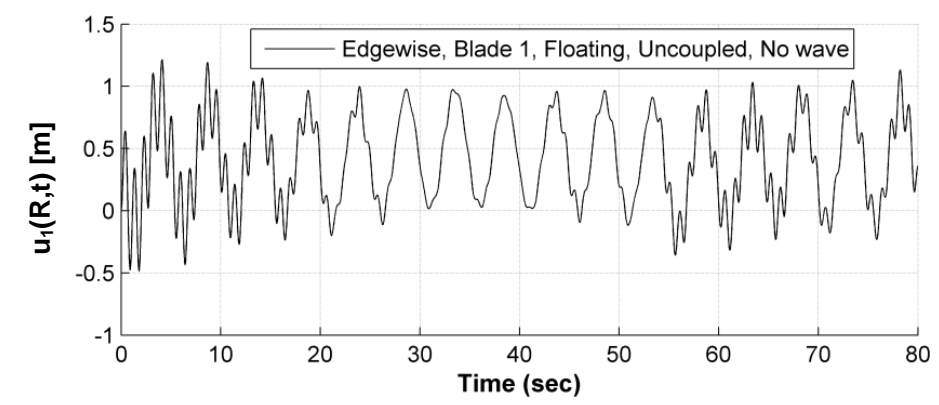

(a) Blade 1, time history

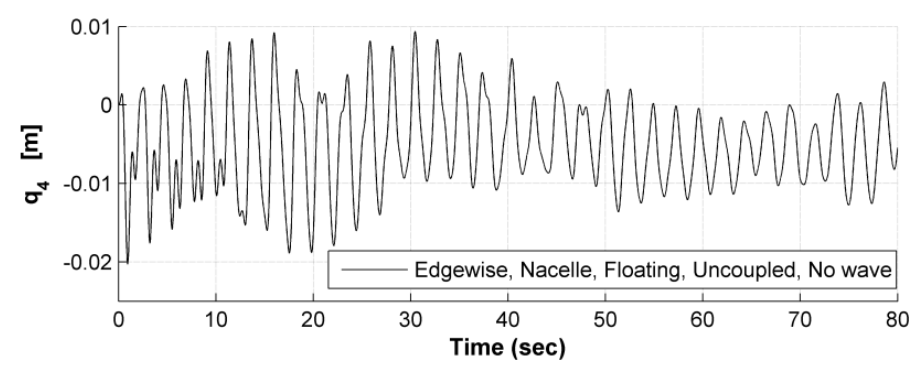

(c) Nacelle, time history

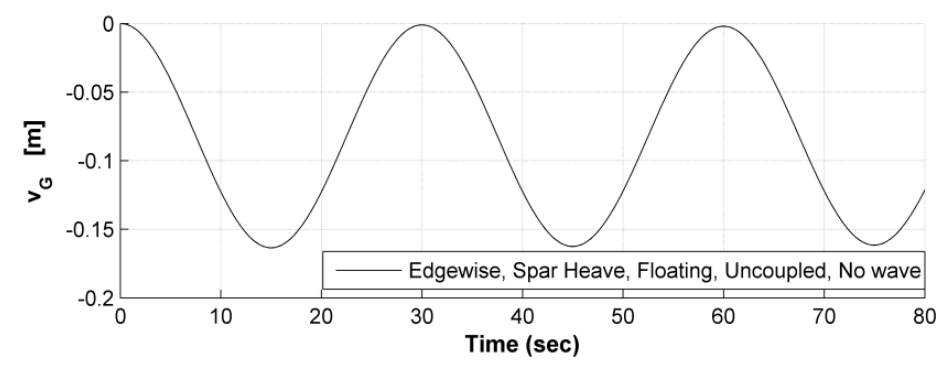

(e) Spar heave, time history

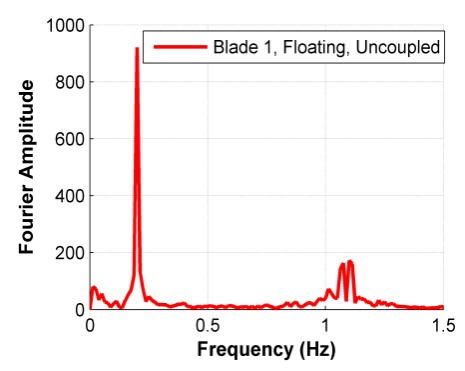

(b) Blade 1, FFT

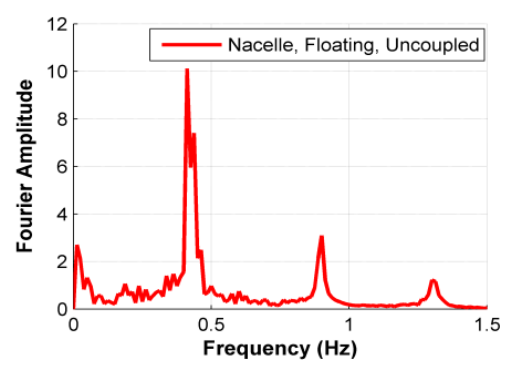

(d) Nacelle, FFT

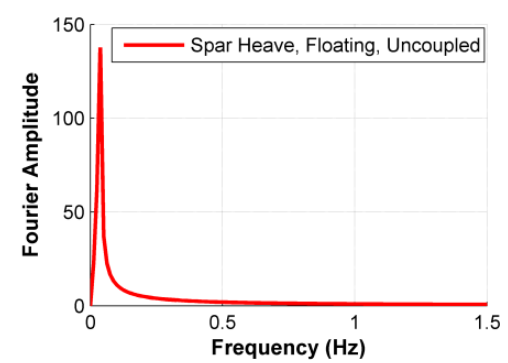

(f) Spar heave, FFT

Fig. 4 Displacements of the 5MW S-FOWT by using uncoupled model in no-wave sea

The amplitude and fundamental frequency of blade and nacelle displacements of the uncoupled S-FOWT in Figs. 4(a)-4(d) are similar to those of the FBWT in Figs. 3(a)-3(d); the impact of spar dynamics on the blades and nacelle response in no-wave sea is therefore not significant. There are reductions in amplitude at higher frequency for the S-FOWT blade displacements as shown in Fig. 4(b) and increase in amplitude at lower frequency for the S-FOWT nacelle displacement as shown in Fig. 4(d), as compared to the FBWT. These can be attributed to the flexibility of FOWT foundation where the lower frequency components of responses are more excited than the higher ones.

\subsection{Response comparisons of uncoupled/ coupled models of S-FOWT in no-wave sea}

The amplitude and frequency content of blade and nacelle displacements of the uncoupled S-FOWT in Figs. 4(a)-4(d) are similar to those of the coupled S-FOWT in Figs. 5(a)-5(d); the impact of the coupling with spar motions on quantities such as the blade and nacelle displacements in no-wave sea is therefore not significant. There is increase in amplitude at lower frequency components of nacelle displacement as shown in Fig. 5(d). 


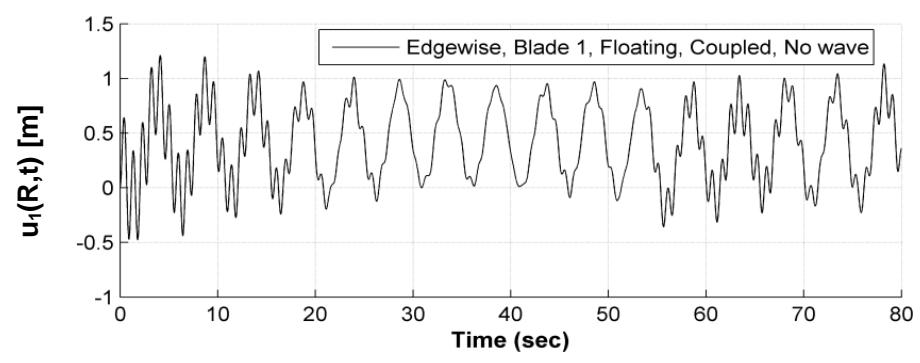

(a) Blade 1, time history

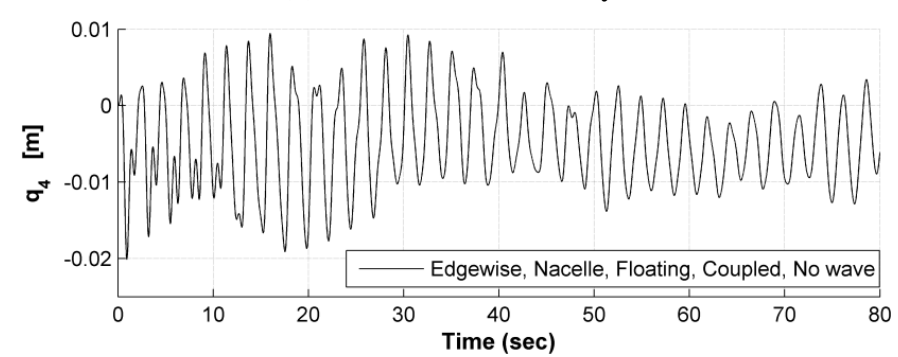

(c) Nacelle, time history

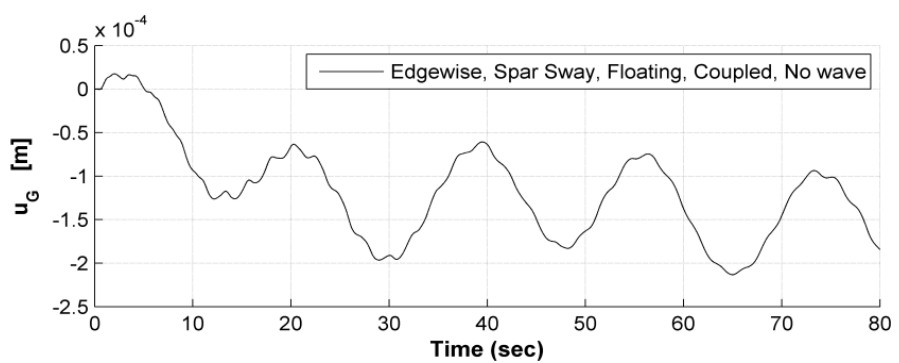

(e) Spar sway, time history

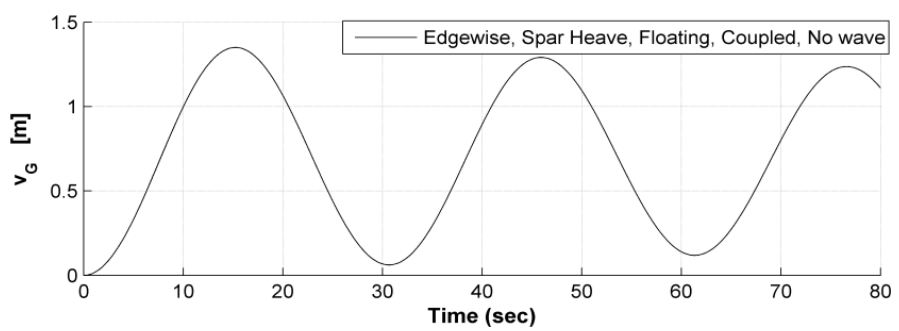

(g) Spar heave, time history

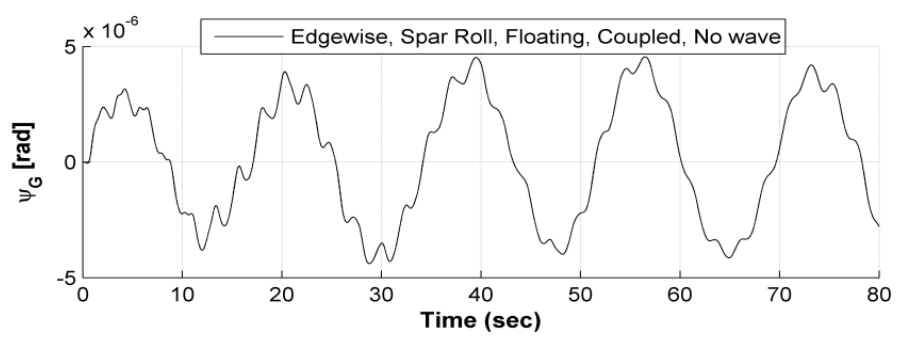

(i) Spar roll, time history

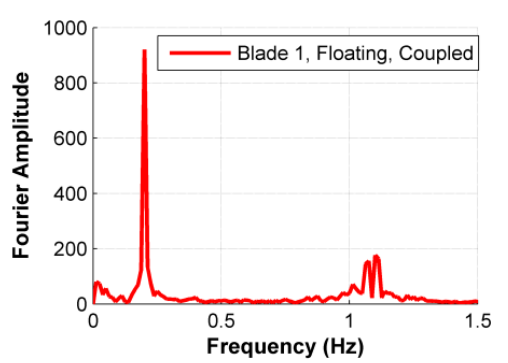

(b) Blade 1, FFT

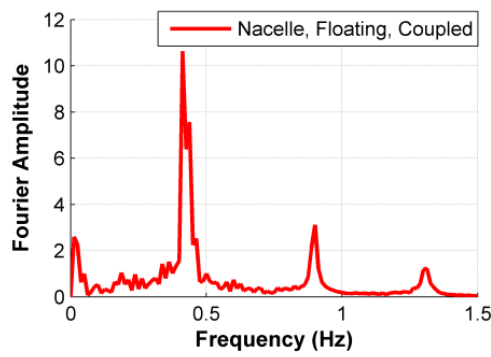

(d) Nacelle, FFT

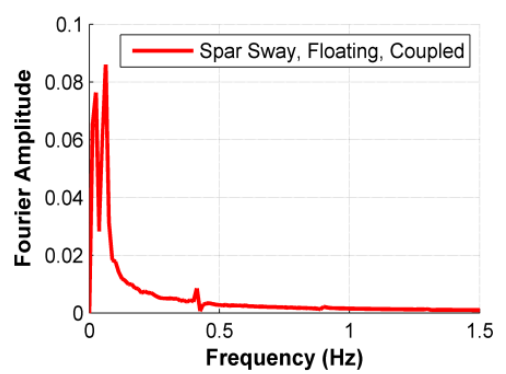

(f) Spar sway, FFT

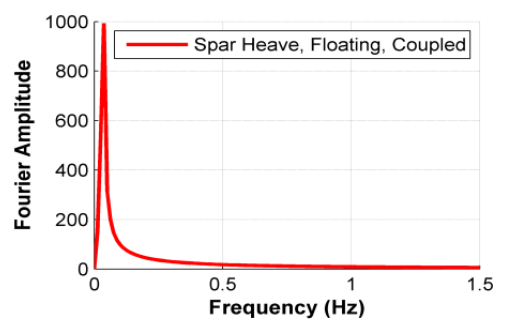

(h) Spar heave, FFT

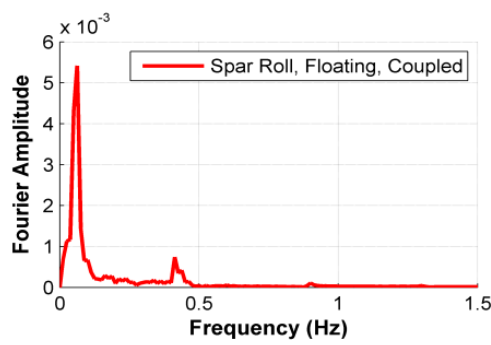

(j) Spar roll, FFT

Fig. 5 Displacements of the 5MW S-FOWT by using coupled model in no-wave sea 


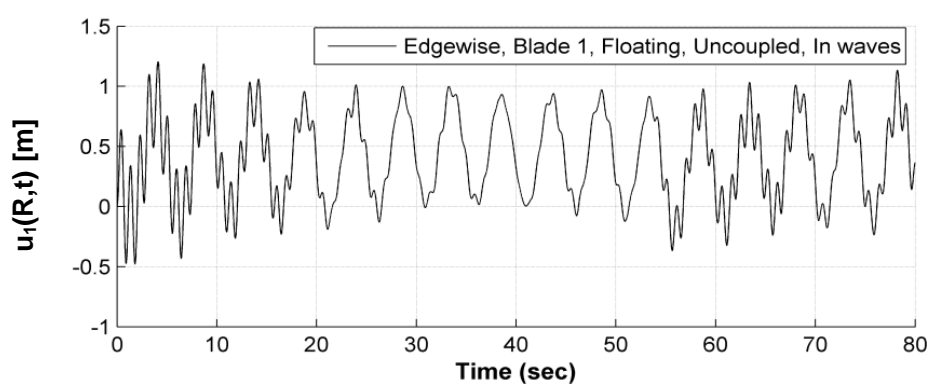

(a) Blade 1, time history

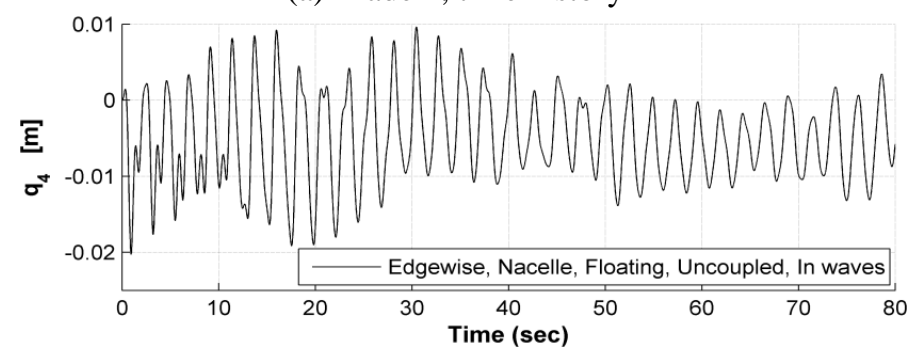

(c) Nacelle, time history

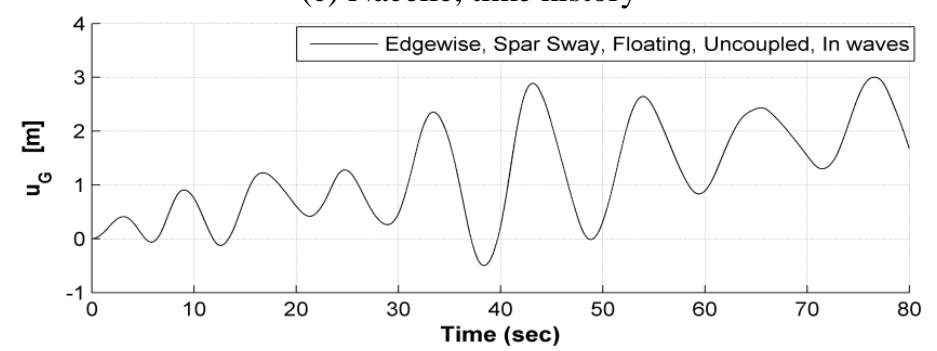

(e) Spar sway, time history

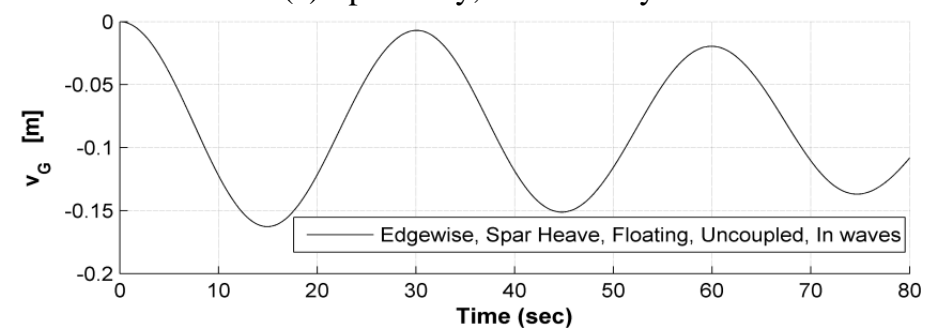

(g) Spar heave, time history

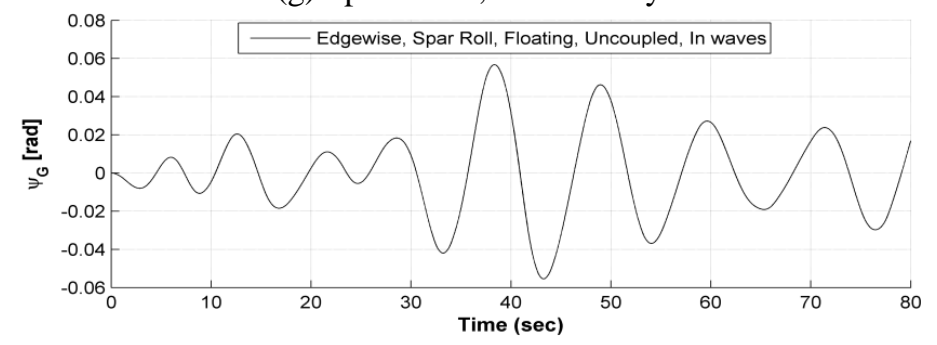

(i) Spar roll, time history

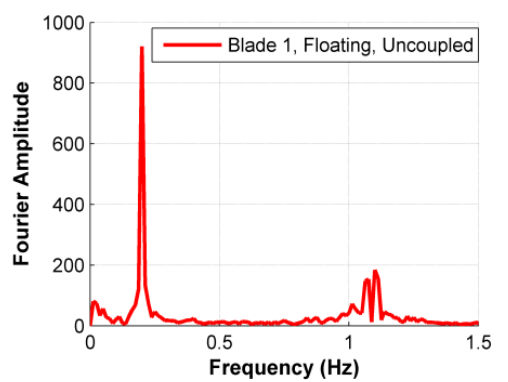

(b) Blade 1, FFT

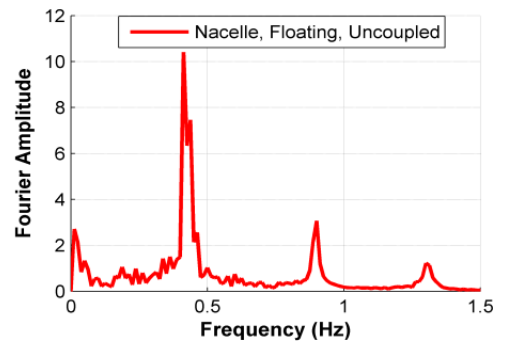

(d) Nacelle, FFT

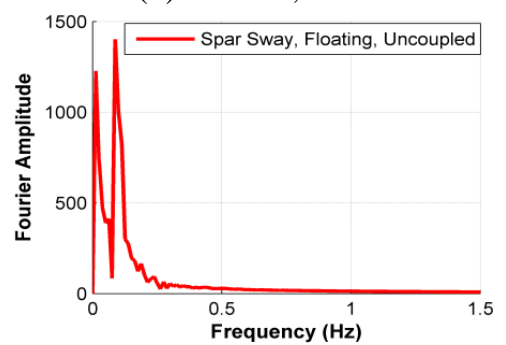

(f) Spar sway, FFT

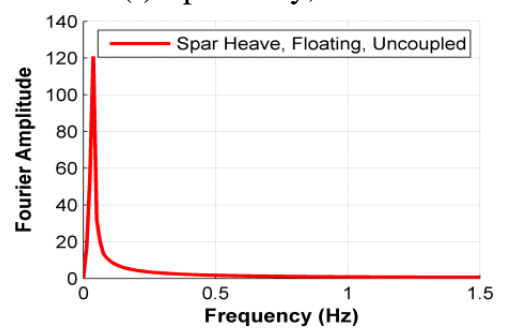

(h) Spar heave, FFT

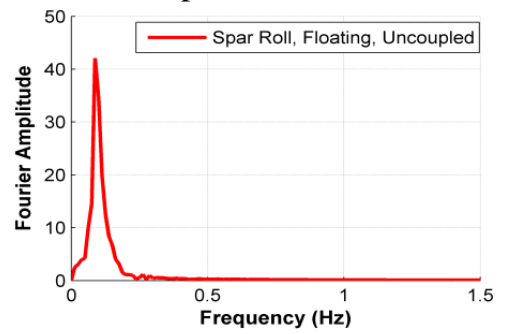

(j) Spar roll, FFT

Fig. 6 Displacements of S-FOWT by using uncoupled model with waves, Eqs. (34) and (35) 
More attention should be paid at the spar motions. There are considerable sway and roll displacements of the coupled S-FOWT model as shown in Figs. 5(e), 5(f), 5(i) and 5(j) even though no waves are considered. Especially, the response amplitude and Fourier amplitude of the spar heave displacements are much larger than those of the uncoupled S-FOWT model. The blade-nacelle-spar couplings are seen to contribute significantly to the spar response in no-wave sea.

\subsection{Response comparisons of uncoupled and coupled models of S-FOWT with waves}

The amplitude and frequency content of the blade displacements of the uncoupled S-FOWT in Figs. 6(a) and 6(b) are similar to those of the coupled S-FOWT in Figs. 7(a) and 7(b); the impact of the coupling with spar motions on the blade responses in waves is also not significant. However, there are differences in both amplitude and frequency content of the nacelle response. The nacelle displacement of the uncoupled model with waves in Figs. 6(c) and 6(d) is as small as $0.02 \mathrm{~m}$; its time history and frequency content are similar to those of the response of the model without the wave loading. The effect of wave loads on the nacelle is consequently not reflected in the uncoupled model. On the contrary, the nacelle displacement of the coupled model with wave loading shown in Fig. 7(c) are as large as $2.2 \mathrm{~m}$ and contains contribution mainly from with frequency components lower than $0.15 \mathrm{~Hz}$. The coupled model is shown to capture the effect of waves on the nacelle response.

There are also significant differences in magnitude of the spar displacements between the coupled and uncoupled models. The maximum spar sway of the uncoupled model as shown in Fig. 6(e) is about $3 \mathrm{~m}$ whereas that of the coupled model as shown in Fig. 7(e) is smaller and is about 1.4 $\mathrm{m}$. The maximum spar heave of the uncoupled model as shown in Fig. $6(\mathrm{~g})$ is about $0.15 \mathrm{~m}$ whereas that of the coupled model presented in Fig. 7(g) is larger and is about $1.2 \mathrm{~m}$. The maximum spar roll of the uncoupled model as shown in Fig. 6(i) is about $0.05 \mathrm{rad}$ whereas that of the coupled model shown in Fig. 7(i) is smaller and is about $0.01 \mathrm{rad}$. The impact of the blade-nacelle-spar coupling is therefore seen to be significant for the nacelle and the spar responses under wave loading.

\subsection{Response comparisons of S-FOWT in no-wave sea and with waves}

The influence of wave loading on the nacelle, spar sway and roll displacements depends on the modeling of S-FOWT and is discussed in Sections 9.1-9.3. However, the influence of the wave loading simulated in this paper on the blade edgewise displacement and spar heave displacement are insignificant. In the numerical examples, the wind conditions corresponding to the no-wave sea and to the sea with waves are unrealistically assumed to be identical in order to investigate the effect of wave loading on the response of the S-FOWT.

Comparing Figs. 4(a) and 4(b) with Figs. 6(a) and 6(b), and Figs. 5(a) and 5(b) with Figs. 7(a) and 7(b), no difference is observed in the blade edgewise displacements between the two cases of wave loading. The spar heave displacement is also not considerably increased when wave loading is considered as shown in Figs. 4(e), 4(f), 6(e) and 6(f) for the uncoupled model, and in Figs. 5(e), 5(f), 7(e) and 7(f) for the coupled model. However, in absence of the wave loading, the spar heave displacements are damped out at a slower rate and the peak Fourier amplitudes are slightly larger as compared to the responses with wave loading. The faster decay of heave displacement under wave loading can be attributed to the larger radiation damping due to fluid motion. 


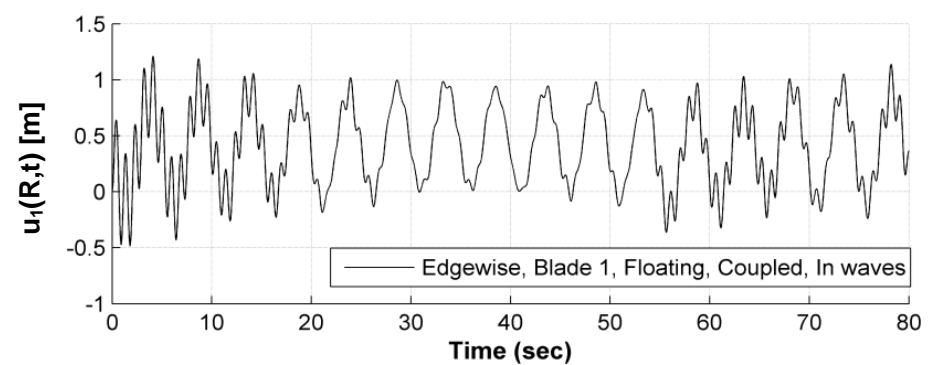

(a) Blade 1, time history

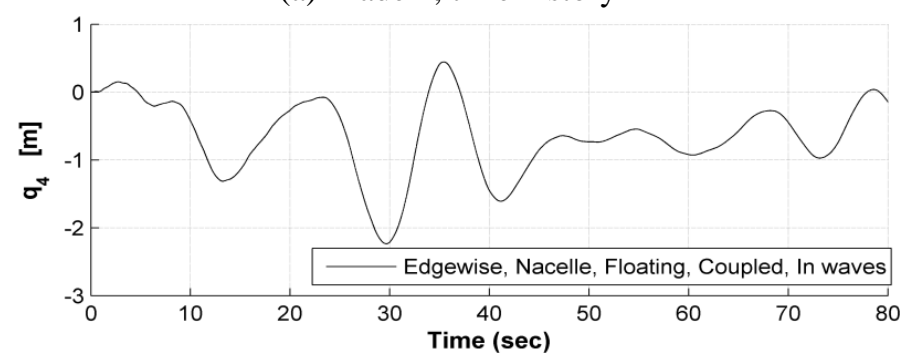

(c) Nacelle, time history

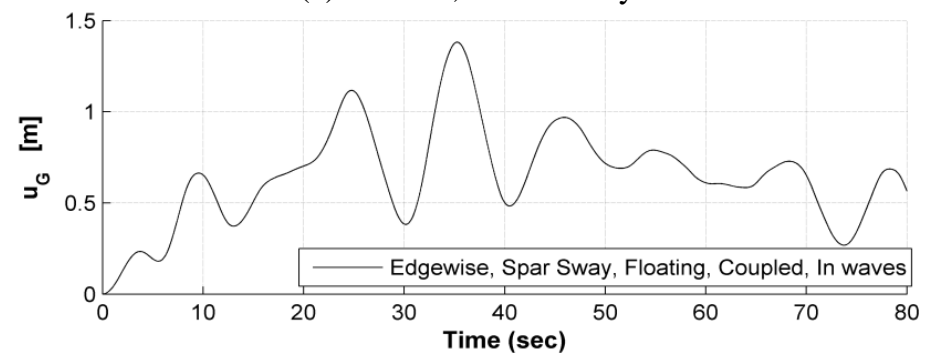

(e) Spar sway, time history

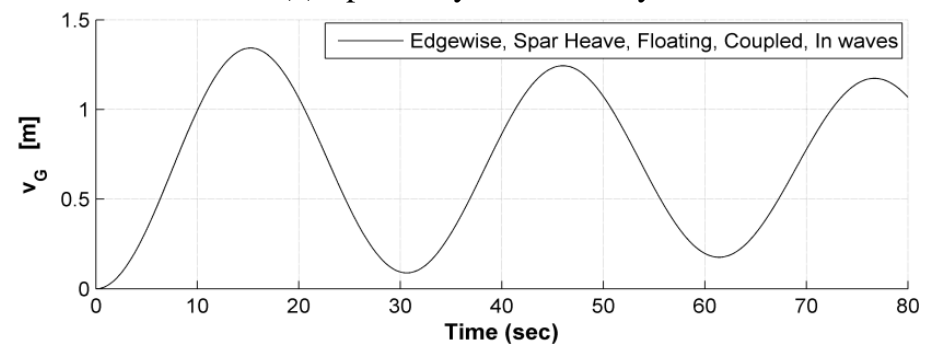

(g) Spar heave, time history

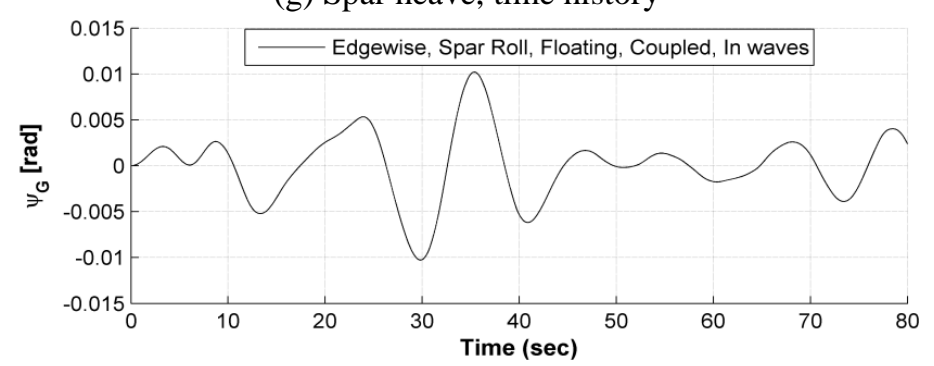

(i) Spar roll, time history

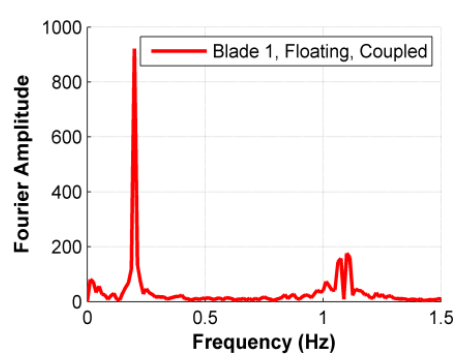

(b) Blade 1, FFT

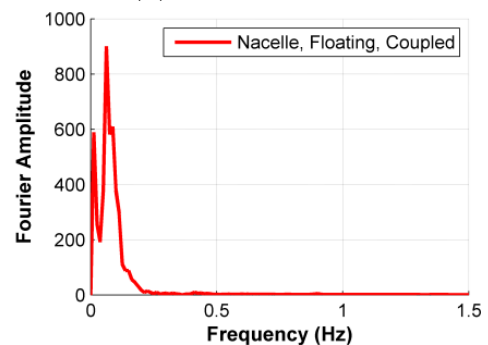

(c) Nacelle, FFT

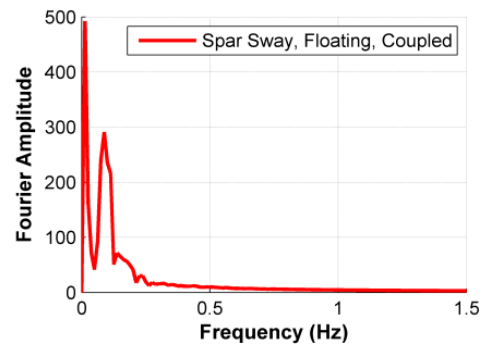

(f) Spar sway, FFT

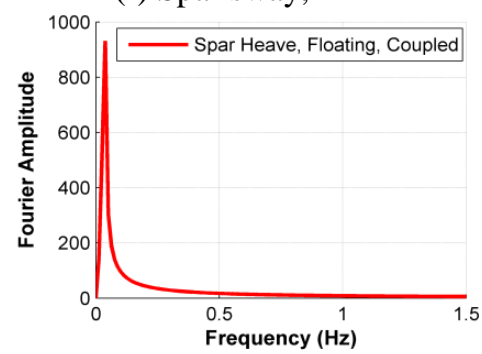

(h) Spar heave, FFT

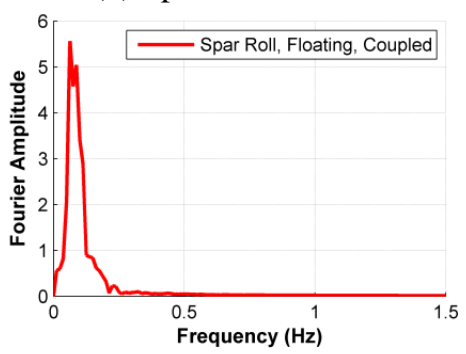

(j) Spar roll, FFT

Fig. 7 Displacements of S-FOWT by using coupled model with waves, Eqs. (36) and (37) 


\section{Conclusions}

The derived formulation of the proposed coupled model for a spar-type floating offshore wind turbine shows that the vibrations of the blades, nacelle, tower, and spar are coupled in all degrees of freedom and in all components: inertial, dissipative and elastic. The gravitational loads and the aerodynamic loads also contain coupling terms between the blades and the spar. The solution to the proposed discrete model for the complete system with the coupling is computationally inexpensive in time while the key components of the system are accounted for. The numerical examples show significant impact of the blade-nacelle-spar coupling on the nacelle and spar responses in several of the cases considered.

Comparing to the fixed-base case, there are reductions in response amplitude at higher frequency components of the S-FOWT blade displacements and increase in response amplitude at lower frequency components of the S-FOWT nacelle displacement due to the flexibility of the FOWT foundation. This is due to the reason that the lower frequency component of the responses is more excited than the higher ones. In the case with no wave loading, the coupling contributes significantly to the spar response but not to the nacelle response. In the case with the wave loading included, the impact of blade-nacelle-spar coupling is significant on the response of both the nacelle and the spar. The coupled model is shown to capture the effect of waves on nacelle response whereas the uncoupled model does not.

\section{Acknowledgements}

The research leading to the results in this paper has received funding from the European Community's Seventh Framework Programme [FP7/ 2007-2013] under the Marie Curie ITN project SYSWIND, Grant No. PITN-GA-2009-238325. The authors are grateful for the support.

\section{References}

Abdel Raheem, S.E. (2013), "Nonlinear response of fixed jacket offshore platform under structural and wave loads", Coupled Syst. Mech., 2(1), 111-126.

Basu, B., Staino, A. and Dinh, V.N. (2012), "Vibration of wind turbines under seismic excitations", Proceedings of the 5th Asian-Pacific symposium on structural reliability and its applications, Paper No. 403, Singapore, May, DOI: 10.3850/978-981-07-2219-7_P403.

Colwell, S. and Basu, B. (2009), "Tuned liquid column dampers in offshore wind turbines for structural control”, Eng. Struct., 31(2), 358-368, DOI:10.1016/j.engstruct.2008.09.001

Dinh, V.N. and Basu, B. (2013), "On the modelling of spar-type floating offshore wind turbines", Key Eng. Mat., 569-570, 63-643, DOI: 10.4028/www.scientific.net/KEM.569-570.636.

Faltinsen, O.M. (1990), Sea loads on ships and offshore structures, Cambridge University Press, UK.

Hansen, M.H. (2003), "Improved modal dynamics of wind turbines to avoid stall-induced vibrations", Wind Energy, 6(2), 179-195, DOI:10.1002/we.79.

Haslum, H.A. (1999) "Alternative shape of spar platforms for use in hostile areas", Proceedings of the offshore technology conference, Houston, Texas, May.

Henderson, A.R. and Patel, M.H. (2003), "On the modelling of a floating offshore wind turbine", Wind Energy, 6(1), 53-86, DOI: 10.1002/we.83. 
IEC 61400-3 (2006), Wind turbines - part 3: design requirements for offshore wind turbines, International Electrotechnical Commission (IEC).

Jain, R.K. (1980), "A simple method of calculating the equivalent stiffnesses in mooring cables", Appl. Ocean Res., 2(3), 139-142, DOI: 10.1016/0141-1187(80)90006-1.

Johannessen, K., Meling, T.S. and Haver, S. (2001), "Joint distribution for wind and waves in the northern North Sea", Proceedings of the international offshore and polar engineering conference, Cupertino, CA, USA.

Jonkman, J.M. (2007), Dynamics modelling and loads analysis of an offshore floating wind turbine, Technical Report NREL/TP-500-41958, NREL, Golden, CO, USA.

Jonkman, J.M., Butterfield, S., Musial, W. and Scott, G. (2009), Definition of a 5-MW reference wind turbine for offshore system development, Technical Report NREL/TP-500-38060, Golden, CO.

Jonkman, J.M. (2010), Definition of the floating system for Phase IV of OC3, Technical Report NREL/TP-500-47535, NREL, Golden, CO, USA.

Kallesøe, B.S., Larsen, T.J., Paulsen, U.S., Køhler, A., Dixen, F.H., Mørch, C.B., Kringelum, J. and Hansen, H.F. (2011), Aero-hydro-elastic simulation platform for wave energy systems and floating wind turbines, Report Ris $\varnothing-\mathrm{R}-1767(\mathrm{EN})$, Technical University of Denmark.

Karimirad, M. and Moan, T. (2011), "Extreme dynamic structural response analysis of catenary moored spar wind turbine in harsh environmental conditions", J. Offshore Mech. Arct., 133(4), 1-13, DOI: $10.1115 / 1.4003393$

Karimirad, M. and Moan, T. (2012), "Wave- and wind-induced dynamic response of catenary moored spar wind turbine", J. Waterw. Port C-ASCE, 138(1), 9-20, DOI: 10.1061/(ASCE)WW.1943-5460.0000087.

Matsukuma, H. and Utsunomiya, T. (2008), "Motion analysis of a floating offshore wind turbine considering rotor rotation”, IES J. Part A: Civil. Struct. Eng., 1(4), 268-279, DOI: 10.1080/19373260802401702.

Nielson, F.G., Hanson, T.D. and Skaare, B. (2006), "Integrated dynamic analysis of floating offshore wind turbines", Proceedings of the 25th international conference on offshore mechanics and arctic engineering, Hamburg, Germany.

Sannasiraj, S.A., Sundar, V. and Sundaravadivelu, R. (1998), "Mooring forces and motion responses of pontoon-type floating breakwaters", Ocean Eng., 25(1), 27-48, DOI: 10.1016/S0029-8018(96).

Sarpkaya, T. and Isaacson, M. (1981), Mechanics of wave forces on offshore structures, Van Nostrand Reinhold, NewYork, USA.

Skaare, B., Hanson, T.D., Nielsen, F.G., Yttervik, R., Hansen, A.M., Thomsen, K. and Larsen, T.J. (2007), "Integrated dynamic analysis of floating offshore wind turbines", Proceedings of European Wind Energy Conference, Milan, Italy, May.

Solberg, T. (2011), Dynamic response analysis of a spar-type floating wind turbine, M.Sc. Thesis, Norwegian University of Science and Technology, Trondheim, Norway.

Staino, A., Basu, B. and Nielsen, S.R.K. (2012), “Actuator control of edgewise vibrations in wind turbine blades", J. Sound Vib., 331(6), 1233-1256, DOI:10.1016/j.jsv.2011.11.003.

Sultania, A. (2010), Reliability analysis of a spar buoy-supported floating offshore wind turbine, M.Sc. Thesis, The University of Texas at Austin, Texas, USA.

Waris, M.B. and Ishihara, T. (2012), "Dynamic response analysis of floating offshore wind turbine with different types of heave plates and mooring systems by using a fully nonlinear model", Coupled Syst. Mech., 1(3), 247-268, DOI: 10.12989/csm.2012.1.3.247. 